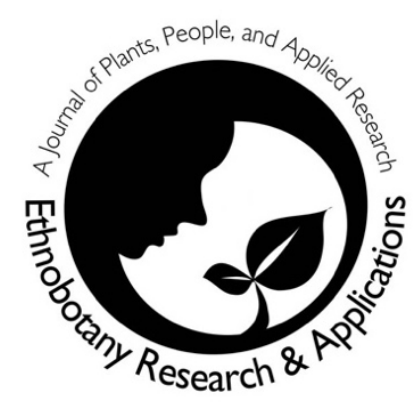

\title{
An ethnobotanical analysis on flora-medicine continuum among the tribal inhabitants of Ratnagiri and Palghar district, Maharashtra, India
}

\author{
Anirban Roy and Supriya Janbandhu
}

\section{Research}

\begin{abstract}
Background: Considering the applicability of wild plants in the pharmaceutical industries, two tribal predominant areas of Maharashtra- Sangameshwar Taluka (Ratnagiri district) and Saphale village (Palghar district) - were chosen to document the local traditional knowledge about medicinal plants.
\end{abstract}

Methods: The ethnomedicinal data were collected through a questionnaire-based survey and extensive personal dialogues adopting the chain sampling referral method with native villagers and Hakims (Traditional healers). The field-based investigations were carried out from September 2017 to April 2018 under the regulatory directives of K.J. Somaiya College of Science and Commerce (Autonomousaffiliated to University of Mumbai).

Results: A total of 51 Traditional Medicinal Plants (TMPs) were documented from the responses of 92 inhabitants in the study areas. The investigators taxonomically categorized these plants into their botanical families, yielding the results- 22 dicot families, 7 monocot families, 2 magnoliids, and 1 pteridophytic family- for the present study. Results revealed that leaves were the most frequently used medicinal part of the documented species and decoction was the most commonly prepared medicinal formulation.
Glossocardia bosvallia, Ricinus communis, and Woodfordia fruticosa - were found to be common in both the study regions. We believed that social factors tend to influence the traditional medicinal knowledge since the same plants were known by different names for treating two unlike ailments. Highest use reports were observed for Terminalia paniculata in Sangameshwar Taluka (Ratnagiri district) and Ampelocissus latifolia in Saphale village (Palghar district). The study realised the fact that both the areas were rich in floral vegetation with interminable floral diversity but remained botanically virgin and unexplored neither for medicinal nor for scientific endeavors.

Keywords: Ethnobotany, Traditional medicinal plants (TMPs), Tribal, Hakims, Maharashtra

\section{Correspondence}

Anirban Roy* and Supriya Janbandhu

Department of Botany, K.J.Somaiya College of Science and Commerce (Autonomous), Affiliated to University of Mumbai, Vidyavihar (E), Mumbai, Maharashtra- 400077, India

*Corresponding Author: anirban.roy@somaiya.edu

Ethnobotany Research \& Applications

20:43 (2020) 


\section{Background}

Ethnobotany- in its wider context- denotes the entire realm of the beneficial relationship between plants and men (Arora 1997). Ethnobotany deals with the complex relationships between (uses of) flora and culture(s), focusing on how plants have been or are used, managed, and perceived in human societies, including plants used for dietary consumption, medicine, divination, cosmetics, dyeing, textiles, building, tools, currency, clothing, rituals, and social life (Rahman 2009). A rational and unswerving effort in this field can yield commendable outcomes of scientific significance worthy for the researches of ethnologists, archaeologists, anthropologists, plantgeographers, botanists, linguists, ecologists, phytochemists, and many more. Being a bridge between nature and humans, it outvies a step ahead of botany, given that it supplies the 'idea' of the basic material for botanical research and study. It then takes us to the utilities of the unmapped medicinal plants, leading a step further to assist the menfolk in the application of that knowledge about the medicinal plants to curb the ailments (Jain 1996). Traditional medicine being employed as a resource for primary health care is an age-old practice in different parts of the world (Budha-Magar et al. 2020). Drugs obtained from plants are believed to be much safer (Katewa et al. 2004) and exhibit a remarkable service in the treatment of various maladies (Siddiqui et al. 2000).

India is home to about $8.6 \%$ of scheduled tribes of the total population (Government of India Census 2011)- the human community subjected to abject poverty and social backwardness due to restricted access to standardized education. They mainly hinge on the traditional medicines to contest against the common ailments due to the absence of expedient health care facilities. The native medicinal plants are the exclusive sources of tribal medicines, the information of which is passed as hierarchical knowledge from their predecessors. The verbal succession of this knowledge of tribal medicine (also known as 'Folk' or 'Indigenous' medicine) is passed from one generation to another without any evidence of written script or digital documentation (Mabogo 2012). According to the World Health Organization (WHO), $80 \%$ of the global population relies upon herbal medicine in their day-to-day lives (Azaizeh et al., 2003, WHO 2012). WHO has also recommended the developing nations to document, preserve, and publicize the ethnobotanical knowledge of medicinal plants (Ministério da Saúde 2005). Kayne (2010) has stressed the role of WHO to mediate regular communication between biomedical scientists, public health authorities, and traditional healers to archive the ethnobotanical knowledge. Previous studies advocated the practice of more than 8000 species of plants and approximately 25,000 folk medicine- based formulations by the Indian tribal and ethnic communities as an integral unit of their healthcare systems (Sharma et al. 2016). The rich biodiversity of India harbors a rich repository of untapped medicinal plants with plenty of botanical awareness that needs to be scientifically explored. More than 1.5 million traditional medical practitioners in India employ medicinal plants for therapeutic, preventive, and restorative purposes- with no or least sideeffects (Sharma et al. 2016).

There have been previous ethno-scientific efforts in India where researchers have attempted to explore the traditional knowledge of inhabitants regarding indigenous medicinal plants and their uses (Chandra 1995, Islam \& Jha 2003, Mitra \& Mukherjee 2005, Chakraborty \& Bhattacharjee 2006, Shanmugam et al. 2009, Kuvar \& Bapat 2010, Das \& Mondal 2013, Yadav \& Khare 2014, Wagh \& Jain 2014, Dileep \& Geetha 2015, Murtem \& Chaudhry 2016, Gupta \& Gupta 2017, Sinha 2017, Esakkimuthu S. et al. 2018). However, it has been observed in past literature that the cavernous depth of ethnobotanical knowledge can be improperly documented for a given region, even after two decades of research (Joshi \& Joshi 2008). In addition to that, Wanjohi et al. 2020 have pointed out that studies based on plant inventories in developing nations are mostly concerned with taxonomic investigations, rather than indigenous knowledge of the inhabitants. Considering the research hitherto, a scientific attempt has been made to document TMPs through the responses of tribal community members from two major tribal predominant districts of MaharashtraRatnagiri and Palghar. Maharashtra is the thirdlargest Indian state by area with a tribal population of 10,510,276 (Government of India Census 2011) rendering this study significant in the light of ethnobotanical research. This study shall also serve as an opportunity to fill the research gaps in the existing ethnobotanical literature since the study sites (in the given districts) were previously unexplored for medicinal plants.

During ethnobotanical field visits in the aforementioned study areas, the investigators encountered an appreciable population of 'tribal and local people' who were expending the wild and semiwild plants for medicinal and other purposes. They were very proficient and skilled in traditional medicine and were prescribing these plant materials to curb different maladies. The objectives of the study were:

A) To make an investigation about the present ethnobotanical status in the study areas of Maharashtra and botanically document the medicinal plants available therein. 
B) To enquire the extent of use of medicinal plants by the tribal (loca)l people.

C) To statistically assess the ethnobotanical information as reported by the folks to deduce legitimate scientific conclusions.

\section{Materials and Methods}

\section{Study Areas}

The research study was conducted in two different sites from two tribal predominant districts of Maharashtra, India.

\section{Saphale Village, Palghar District}

Palghar (Fig. 1) is a district in the northernmost part of the Konkan lowlands of Maharashtra comprising a population of 2,990,116 individuals with $86.58 \%$ of tribal residents (Government of India Census 2011).

It was acknowledged as the $36^{\text {th }}$ district after being separated from Thane district with the maximum density of tribal (Adivasi) people in Maharashtra. Demographically, Palghar district begins with Dahanu at the north and ends at Naigaon towards the south. Geographically, Ulhas basin surrounds Palghar at the southern fringe, while hilly Vaitarna valley is present at the northern periphery together with plateaus and the steep slopes of Sahyadri in the east. There are successions of plateaus present in the center of Palghar district.

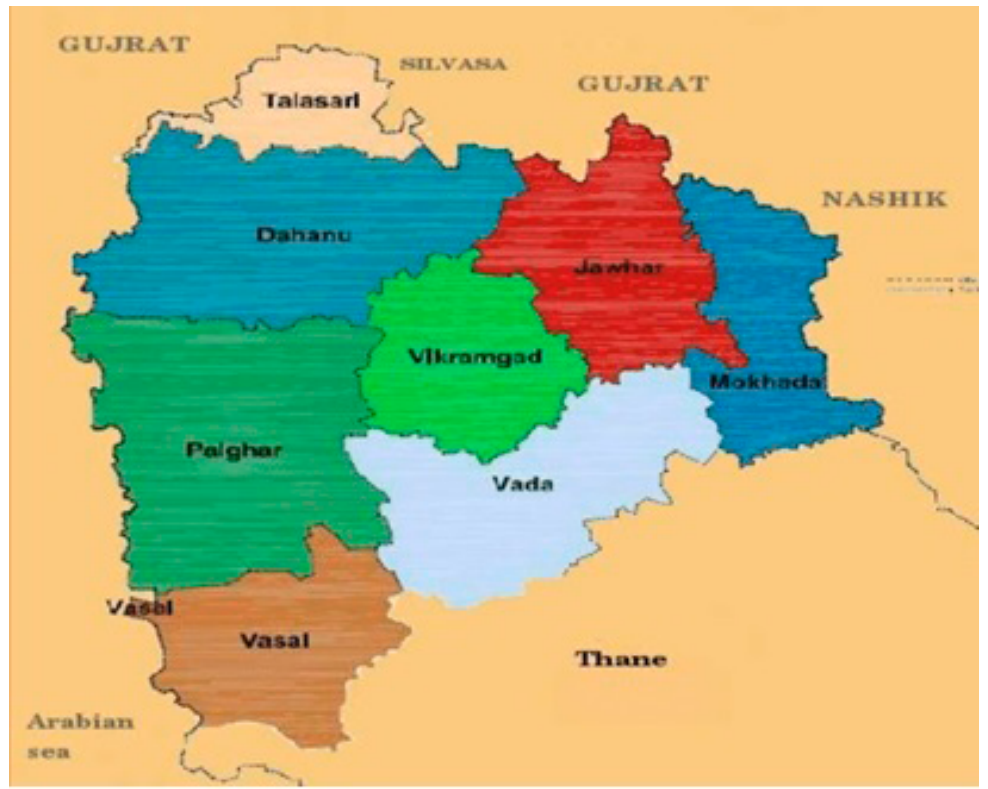

Figure 1. Map of Palghar district, Maharashtra. Image credit: Image Credit: Palghar district map. [Photograph]. (n.d.). Retrieved from: https://www.mapsofindia.com/assemblypolls/maharashtra/palghar.html

The investigators selected Saphale village as the representative site in the Palghar district. The GPS coordinates of Saphale village read as $19^{\circ} 36^{\prime} 15.15^{\prime \prime} \mathrm{N}$ latitude and $72^{\circ} 50^{\prime} 1.76 " \mathrm{E}$ longitude and is present at a height of 65.37 masl. It has a population of 4396 individuals with $51 \%$ male and 49 $\%$ female dwellers (Government of India Census 2011). Located at the north of the Vaitarna river, the village has a majority of Hindu religion devotees with famous Hindu shrines like Kurlai Devi Temple, Charbhuja Temple, Shree Datta Temple, Shiv Sankar Temple, Harbadevi Temple Dongare, and many more. The soil of this region is primarily black, shallow type with $\mathrm{pH}$ ranging from 7.4 to 8.2 containing less clay and silt but rich in organic matter. Saphale village has a tropical climate and experiences heavy precipitation during summers. The average annual temperature and the average rainfall in Saphale are $26.5^{\circ} \mathrm{C}$ and $2101 \mathrm{~mm}$ respectively.

\section{Sangameshwar Taluka, Ratnagiri District}

One of the pristine natural attractions of Western Ghats, Ratnagiri is a district in the southwestern part of Konkan lowlands of Maharashtra comprising a population of 76,239 people (Government of India Census 2011). Geographically, Ratnagiri is located at the coordinate of $16.98^{\circ} \mathrm{N}$ and $73.3^{\circ} \mathrm{E}$ with an average elevation of 11 masl. On the eastern edge, Sahyadri Mountains border Ratnagiri while Sindhudurg district is present towards the southern end. Ratnagiri has a moderate- kind of climatic condition with an average temperature of $23^{\circ} \mathrm{C}$ and average precipitation of $2938.4 \mathrm{~mm}$. It has a port city with the name of Ratnagiri on the western banks of the Arabian Sea coast, which experiences a commendable trade contributing to the economy of the state as well as the nation. 
Sangameshwar Taluka is chosen as the representative site from the Ratnagiri district (Fig. 2). The soil type of this place is primarily laterite soil and coastal alluviums, with laterite soil being largely spread among the two. The soil is saline due to the deposition of sediments by river Shastri and river Sonavi with alkaline $\mathrm{pH}$. Located at the juncture of the two rivers, this place experiences a considerate warm climate with the highest temperature of $39^{\circ} \mathrm{C}$ during summers. It is situated $42 \mathrm{~km}$ towards East from the district headquarters of Ratnagiri and located at an elevation of 37 masl. Sangameshwar Taluka is bounded by Ratnagiri Taluka towards the west, Lanja Taluka towards the south, Chipalun Taluka towards the north, and Patan Taluka towards the East.

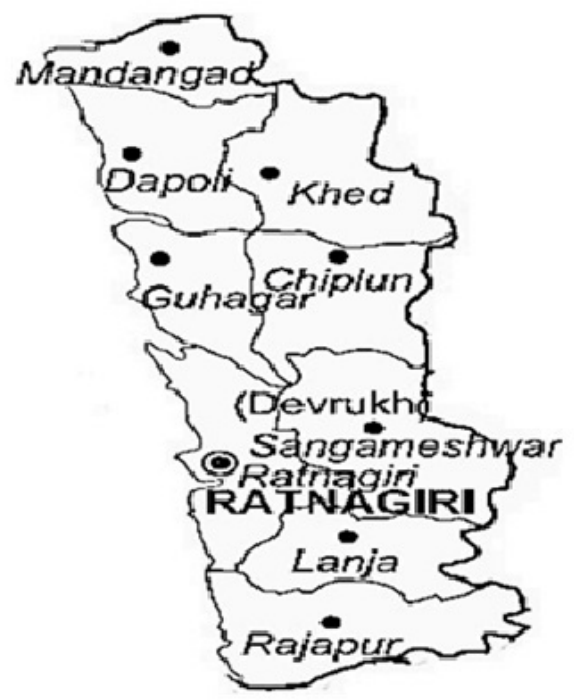

Figure 2. Map of Ratnagiri district, Maharashtra. Image Credit: Ratnagiri district map. [Photograph]. (n.d.). Retrieved from:

https://www.onefivenine.com/india/villag/Ratnagiri

\section{Interviews and Consensus Survey}

The local knowledge of non-institutionally (no formal education) trained Hakims and tribal inhabitants on medicinal flora were documented between September 2017 and April 2018 using a questionnaire-based survey and exhaustive personal dialogues. The interview protocols used for this survey were following the previously published methods (Esakkimuthu et al. 2018, Heinrich et al. 2009, Weckerle et al. 2018) and also with the guidelines of ISE code of ethics for ethnobiological research (International Society of Ethnobiology Code of Ethics- with 2008 additions). This study focused on the local knowledge of Hakims and villagers who learned only through traditional methods of teaching (non-institutional training), practicing and using the plants for a minimum of 5 years, and willing to share their knowledge. Chain referral sampling method (Cavéchia \& Proença 2007, McMillen 2012, Mustafa et al. 2012) was used to recruit the informants- they were selected irrespective of age (above 20 years), gender, education, and community.

The motive behind the survey was cogently elaborated to the participants in simple terms: one or two visits were planned to acquire familiarity in both the districts. The formal interviews were conducted after getting the written consent of informants to participate in this survey (in their colloquial languages). In this way, the local knowledge of 13 non-institutionally trained Hakims and 79 villagers from both the places on medicinal plants were recorded. The open-ended oral questionnaire employed in this survey was divided into two subsequent sections:

(i) the anthropological data related to the demography of the informants such as age, gender, educational qualification, mode of learning traditional medicine, experience, nativity (tribe), residential area, and occupation were enlisted;

(ii) the data regarding the medicinal plants, the criterion for selection of the plants, the ingredients used to prepare these medicines, plant parts used, mode of preparation, illnesses treated with these plant and plant part(s), dosage, combinational aspects and duration of consumption were documented. In the case of combinatorial use of two or more plants, the key medicinal taxa which were perceived to attribute the therapeutic impacts were also recorded.

The investigators along with the informants visited the fields and allied forest areas from where the informants usually bring together the samples, including local shops. The interviews were conducted in the local language Marathi, and at times, in their tribal dialect with the help of a linguistic paraphraser. All interactions were digitally recorded and crossverified to establish a more tangible perception for ambiguous responses. The data were translated into English in the linguistic laboratory of the Institute. Relevant medical terms for the illnesses were assigned by reciprocating the Marathi terminologies to English and the systematic classification of the plants, based on the botanical attributes, was done by a Phytotaxonomist.

\section{Plant Specimens}

The plant specimens- the flowering twigs- were collected and preserved in herbarium sheets at the Department of Botany, K.J. Somaiya College of Science and Commerce, Mumbai. The plant parts that have been mentioned as 'medicinally significant' were also refrigerated in disparate vouchers for documentation. The taxonomic classification of the TMPs was confirmed by a Phytotaxonomist, who 
identified the botanical names for the plant samples. The TMPs were further authenticated using the international plant name index (http://www.ipni.org), the plant list (www.theplantlist.org), the flowers of India (www.flowersofindia.net) and GRIN taxonomy site (http://www.ars-grin.gov/cgibin/npgs/html/queries.pl).

The botanical families were recognized with the help of angiosperm Phylogeny Website (Stevens 2001). The species entries were accompanied along with data on taxonomic position (family), vernacular name, life cycle, life form, and ethnomedicinal uses (Tables 1 and 2). The life form was categorized into herbs, shrubs, climbers, grasses, and trees (annual, biennial, or perennial), according to the system proposed by Raunkiær (1934) and modified by Brown (1977). 5\% sugar solution, added with a pinch of vinegar (an antibacterial agent) was prepared as a preservative for infection-prone plant parts like fruits, flower buds, roots, etc. In the case of trees, the photographs were clicked along with enlisting the physiognomic characters and collection of the leafy and flowering branches for preserving in the herbarium.

\section{Quantification of Data by Ethnobotanical Inventories}

The informants indicated the plants based on their medicinal properties and the type of ailments treated with them, therefore, the key medicinal taxa were selected by the investigators. Both the areas possess unique physiognomic, contour, climatic, and edaphic parameters, and hence, the quantification of data with the help of ethnobotanical inventories reflected diverse outcomes. Based on emic perceptions, Medicinal (Ayurvedic) Botany has defined three types of doshas prevalent in the human body- 'Vata', 'Kapha', and 'Pitta' doshas. The predominant attributes of 'Vata' dosha include the neuropsychiatric and musculoskeletal ailments, while disease manifestation of 'Pitta' dosha includes the gastrointestinal and inflammatory disorders and 'Kapha' dosha include the pulmonary and respiratory diseases (Jayasundar 2010). The following statistical parameters quantified the raw data to discern the relationship between the TMPs and the ailments being referred to be treated by the TMPs:

Use Reports (UR): The collected data were then converted into Use Reports (UR) (Bhat et al. 2013) which can be defined as "Informant (i) refers a plant species(s) for an illness". For example, if two informants mention a species for the treatment of an illness category; it yields two UR(s). URs are, therefore, calculated based on the responses of the tribal informants regarding the medicinal plants.
Informant Consensus Factor $\left(F_{i c}\right)$ : To perceive the potential of the medicinal plants, Trotter and Logan (1986) introduced a method based on the concept of "informant consensus", wherein they compared the URs of a plant for a given ailment with the number of other plants indicated as a remedy to the same ailment. Mathematically, $F_{\text {ic }}$ gives the relationship between the 'number of use reports in each category $\left(n_{u r}\right)$ minus the number of taxa used for that illness $\left(n_{t}\right)$ ' and the 'number of use-reports in each category minus 1' (Heinrich et al. 1998). $F_{\text {ic }}$ is, thus, calculated using the following formula:

$$
\mathrm{F}_{\mathrm{ic}}=\mathrm{n}_{\mathrm{ur}}-\mathrm{n}_{\mathrm{t}} / \mathrm{n}_{\mathrm{ur}}-1
$$

Canales et al. (2005) has used $F_{i c}$ to indicate the agreement of villager's knowledge on the usage of the medicinal plants for a specific ailment. The scale of $F_{i c}$ has a range of 0 to 1 where the highest value of 1 indicates the plant taxa which is used for treating a higher number of ailments and is reported by a large section of individuals. The lowest value of 0 refers to the plant taxa used for the treatment of very few ailments and is randomly chosen by the informants or the informants have insufficient knowledge about the utilities of plant species (AbuIrmaileh \& Afifi 2003, Gazzaneo et al. 2005, Kloutsos 2001, Teklehaymanot 2009). Here, ' 1 ' represents the highest value of informant consensus.

\section{Frequency Citation (FC) and Relative Frequency Citation (RFC):}

Vijayakumar et al. (2016) have explained the concept of RFC as an imperative index for calculating the effectiveness of a TMP. The index is calculated by dividing Frequency Citations (FC) by the total number of informants in the survey $(\mathrm{N})$. FCs are the number of individuals that have mentioned a species as useful; nonetheless, FCs are the measure of the plant's utilities. The scale of RFC ranges from 0 (when nobody considers a plant as useful) to 1 (when all the informants mention it as useful) (Rehman et al. 2017)

RFC is, therefore, calculated using the following formula:

$$
\mathrm{RFC}=\mathrm{FCs} / \mathrm{N}
$$

\section{Use-Value (UV)}

Gazzaneo et al. 2005 demonstrated the Use Value as the relative importance of locally known plants.

$$
U V=\sum U_{i} / N
$$

where $U_{i}$ is the number of uses mentioned by each informant for a given species and $\mathrm{N}$ is the total number of informants in the survey. 
Table 1: List of Plant taxa cited by tribal inhabitants of Sangameshwar Taluka, Ratnagiri district of Maharashtra for preparing medicinal foods (The abbreviations associated with the ailment(s) are described in Tab.- 3$)^{*}$ : voucher specimen not available

\begin{tabular}{|c|c|c|c|c|c|c|c|c|}
\hline Botanical name & $\begin{array}{l}\text { Vernacular } \\
\text { names }\end{array}$ & Habit & $\begin{array}{l}\text { Parts } \\
\text { used }\end{array}$ & $\begin{array}{l}\text { Ailments } \\
\text { treated }\end{array}$ & Vouchers & Herbal recipes & $\begin{array}{c}\text { Applications } \\
\text { to the } \\
\text { patients }\end{array}$ & $\begin{array}{c}\text { Used in a mix } \\
\text { with (solvents or } \\
\text { other plant parts) }\end{array}$ \\
\hline \multicolumn{9}{|l|}{ Acanthaceae } \\
\hline Adhatoda vasica Nees & Adulasha & Shrub & Leaves & Asthma (RED) & AR Sa 04 & $\begin{array}{l}\text { Smoke of the dried } \\
\text { leaves is inhaled by } \\
\text { the patients. }\end{array}$ & Inhalation & - \\
\hline Asteracantha longifolia Nees & Kateri & Shrub & Leaves & Itching (DED) & AR Sa 05 & $\begin{array}{l}\text { Leaves are made into } \\
\text { a paste and applied. }\end{array}$ & Topical & - \\
\hline \multicolumn{9}{|l|}{ Anacardiaceae } \\
\hline Anacardium occidentale $\mathrm{L}$. & Kaju & Tree & Bark & $\begin{array}{l}\text { Itching, } \\
\text { Rashes, } \\
\text { Irritation } \\
\text { (DED) }\end{array}$ & AR Sa 12 & $\begin{array}{l}\text { Bark extract of is } \\
\text { applied to the ailing } \\
\text { site of skin. }\end{array}$ & Topical & $\begin{array}{l}\text { Bark extracts of } \\
\text { Jambhul and Kaju } \\
\text { are mixed together. }\end{array}$ \\
\hline Mangifera indica L. & Amba & Tree & Bark & $\begin{array}{l}\text { Inflammation } \\
\text { (IND) }\end{array}$ & AR Sa 19 & $\begin{array}{l}\text { Bark extracts of } \\
\text { Mango and Pangera } \\
\text { are mixed to apply on } \\
\text { epithelial swelling. }\end{array}$ & Topical & $\begin{array}{l}\text { Mixed with bark } \\
\text { extract of Pangera. }\end{array}$ \\
\hline \multicolumn{9}{|l|}{ Asparagaceae } \\
\hline $\begin{array}{c}\text { Chlorophytum tuberosum } \\
\text { (Roxb.) Baker }\end{array}$ & $\begin{array}{l}\text { Safed Musli, } \\
\text { Kuli }\end{array}$ & Herb & Tuber & Insanity (MED) & AR Sa 03 & $\begin{array}{c}\text { Tubers are boiled and } \\
\text { then given to the } \\
\text { mentally deranged } \\
\text { patients. }\end{array}$ & Oral & Water \\
\hline \multicolumn{9}{|l|}{ Combretaceae } \\
\hline $\begin{array}{c}\text { Terminalia bellerica (Gaertn.) } \\
\text { Roxb. }\end{array}$ & $\begin{array}{l}\text { Baheda, } \\
\text { Goting }\end{array}$ & Tree & Bark & $\begin{array}{l}\text { Pediatric } \\
\text { helminthic } \\
\text { infections } \\
\text { (NPD) }\end{array}$ & * & $\begin{array}{l}\text { Oral administration of } \\
\text { stem infusion to the } \\
\text { babies. }\end{array}$ & Oral & Water \\
\hline Terminalia paniculata Roth. & Kinjal & Tree & Leaves & Injury (BIW) & AR Sa 25 & $\begin{array}{c}\text { Fresh leaves are } \\
\text { taken and rubbed } \\
\text { against the laceration. }\end{array}$ & Topical & - \\
\hline \multicolumn{9}{|l|}{ Compositae } \\
\hline Glossocardia bosvallia (L.f.) DC. & $\begin{array}{l}\text { Patharsuva, } \\
\text { Pithari }\end{array}$ & Herb & $\begin{array}{l}\text { Whole } \\
\text { plant }\end{array}$ & $\begin{array}{c}\text { Sores and } \\
\text { wounds (BIW) }\end{array}$ & AR Sa 16 & $\begin{array}{l}\text { Paste of the whole } \\
\text { plant is used against } \\
\text { physical wounds. }\end{array}$ & Topical & - \\
\hline \multicolumn{9}{|l|}{ Cyperaceae } \\
\hline Cyperus rotundus $\mathrm{L}$. & Nagarmotha & Herb & Rhizome & $\begin{array}{l}\text { Sun Stroke } \\
\text { (SUS) }\end{array}$ & AR Sa 06 & $\begin{array}{l}\text { Decoction of rhizomes } \\
\text { is administered to the } \\
\text { patients. }\end{array}$ & Oral & Water \\
\hline \multicolumn{9}{|l|}{ Euphorbiaceae } \\
\hline Ricinus communis $\mathrm{L}$. & Murud & Tree & Leaves & Fever (PYR) & AR Sa 17 & $\begin{array}{l}\text { Fresh leaves are } \\
\text { taken, mixed with }\end{array}$ & Oral & Ghee \\
\hline
\end{tabular}




\begin{tabular}{|c|c|c|c|c|c|c|c|c|}
\hline & & & & & & $\begin{array}{l}\text { ghee and served to } \\
\text { the patients. }\end{array}$ & & \\
\hline \multicolumn{9}{|l|}{ Gesneriaceae } \\
\hline $\begin{array}{c}\text { Rhynchoglossum obliquum } \\
\text { Blume }\end{array}$ & Neel & Herb & $\begin{array}{l}\text { Leaves \& } \\
\text { Stems }\end{array}$ & $\begin{array}{c}\text { Insect/ Snake } \\
\text { bites, } \\
\text { Poisoning } \\
\text { (BIW) }\end{array}$ & AR Sa 01 & $\begin{array}{l}\text { Paste of leaves \& } \\
\text { stems applied together } \\
\text { at the poisoned site. }\end{array}$ & Topical & - \\
\hline \multicolumn{9}{|l|}{ Lamiaceae } \\
\hline Vitex negundo L. & Nirgund & Tree & Leaves & $\begin{array}{l}\text { Wound and } \\
\text { bites (BIW) }\end{array}$ & * & $\begin{array}{l}\text { Leaf extract added } \\
\text { with salt is applied. }\end{array}$ & Topical & Salt \\
\hline Vitex trifolia L. & Katarnirgundi & Shrub & Leaves & $\begin{array}{l}\text { Inflammation } \\
\text { (IND) }\end{array}$ & AR Sa 11 & $\begin{array}{l}\text { Decoction is prepared } \\
\text { and applied at the site } \\
\text { of protuberance. }\end{array}$ & Topical & Water \\
\hline \multicolumn{9}{|l|}{ Leguminosae } \\
\hline Cassia tora $\mathrm{L}$. & Takala & Shrub & Leaves & $\begin{array}{l}\text { Nail pain in } \\
\text { toes, injury } \\
\text { (BIW) }\end{array}$ & AR Sa 14 & $\begin{array}{l}\text { Leaves are made into } \\
\text { paste and applied. }\end{array}$ & Topical & - \\
\hline Dolichos trilobus L. & Ranval & Climber & Roots & $\begin{array}{l}\text { Leucorrhoea } \\
\text { (GYD) }\end{array}$ & AR Sa 07 & $\begin{array}{l}\text { Oral administration of } \\
\text { root infusion to the } \\
\text { female patients. }\end{array}$ & Oral & Water \\
\hline Erythrina indica Lam. & Pangera & Tree & Bark & $\begin{array}{l}\text { Inflammation } \\
\text { (IND) }\end{array}$ & AR Sa 02 & $\begin{array}{l}\text { Bark extracts of } \\
\text { Mango and Pangera } \\
\text { are mixed to apply on } \\
\text { epithelial swelling. }\end{array}$ & Topical & $\begin{array}{l}\text { Mixed with bark } \\
\text { extract of Mango. }\end{array}$ \\
\hline \multicolumn{9}{|l|}{ Lythraceae } \\
\hline Woodfordia fruticosa (L.) Kurz. & Dhausad & Shrub & Leaves & $\begin{array}{c}\text { Injury and } \\
\text { insect/ snake } \\
\text { bites (BIW) }\end{array}$ & AR Sa 08 & $\begin{array}{c}\text { Leaves dried and } \\
\text { powdered at the site of } \\
\text { abrasion. }\end{array}$ & Topical & - \\
\hline \multicolumn{9}{|l|}{ Malvaceae } \\
\hline Ceiba pentandra (L.) Gaertn. & Samali, Kapok & Tree & Bark & $\begin{array}{c}\text { Diabetes } \\
\text { Mellitus } \\
\text { Type- } 2 \text { (END) }\end{array}$ & * & $\begin{array}{l}\text { Bark decoction is } \\
\text { prepared and drunk. }\end{array}$ & Oral & Water \\
\hline Urena lobata L. & Vana bhindi & Shrub & Roots & $\begin{array}{l}\text { Rheumatism } \\
\text { (Arthritis) of } \\
\text { Bones (SKA) }\end{array}$ & AR Sa 26 & $\begin{array}{l}\text { Juice of roots is drunk } \\
\text { by patients of } \\
\text { rheumatism. }\end{array}$ & Oral & Water \\
\hline \multicolumn{9}{|l|}{ Moraceae } \\
\hline Artocarpus hirsutus Lam. & Aayin & Tree & Bark & $\begin{array}{c}\text { Livestock } \\
\text { bleeding (VD) }\end{array}$ & AR Sa 10 & $\begin{array}{l}\text { Juice from bark is } \\
\text { applied at injury. }\end{array}$ & Topical & - \\
\hline Ficus racemosa $\mathrm{L}$. & Umber & Tree & Roots & Tonsils (GEH) & AR Sa 13 & $\begin{array}{l}\text { Whole root is chewed } \\
\text { to combat tonsillitis. }\end{array}$ & Oral & - \\
\hline \multicolumn{9}{|l|}{ Myrtaceae } \\
\hline Psidium guajava $\mathrm{L}$. & Peru & Tree & Leaves & $\begin{array}{l}\text { Dental } \\
\text { problems } \\
\text { (DEND) }\end{array}$ & AR Sa 18 & $\begin{array}{l}\text { The leaves are taken } \\
\text { and chewed. }\end{array}$ & Oral & - \\
\hline Syzigium cumini (L.) Skeels & Jambhul & Tree & Bark & $\begin{array}{l}\text { Skin problems } \\
\text { (DED) }\end{array}$ & AR Sa 27 & $\begin{array}{c}\text { Bark extract of } \\
\text { Jambhul (Black Plum) }\end{array}$ & Topical & $\begin{array}{l}\text { Mixed with bark } \\
\text { extract of Cashew }\end{array}$ \\
\hline
\end{tabular}




\begin{tabular}{|c|c|c|c|c|c|c|c|c|}
\hline & & & & & & $\begin{array}{l}\text { and Cashew mixed to } \\
\text { treat skin infections. }\end{array}$ & & \\
\hline \multicolumn{9}{|l|}{ Phyllanthaceae } \\
\hline Phyllanthus emblica L. & Awla, Amla & Tree & Stem & $\begin{array}{c}\text { Snake bite, } \\
\text { Scorpion bite } \\
\text { (BIW) }\end{array}$ & AR Sa 28 & $\begin{array}{l}\text { Oral administration of } \\
\text { stem infusion to the } \\
\text { poisoned patients. }\end{array}$ & Oral & Water \\
\hline \multicolumn{9}{|l|}{ Piperaceae } \\
\hline Piper betle L. & Paan & Climber & Leaves & $\begin{array}{c}\text { Constipation } \\
\text { (GID) }\end{array}$ & AR Sa 29 & $\begin{array}{c}\text { Whole leaf is chewed } \\
\text { against teeth. }\end{array}$ & Oral & - \\
\hline \multicolumn{9}{|l|}{ Poaceae } \\
\hline Panicum miliaceum L. & Bhagar & Herb & Grains & $\begin{array}{l}\text { Digestive } \\
\text { disorders } \\
\text { (GID) }\end{array}$ & AR Sa 30 & $\begin{array}{c}\text { Cooked grains are } \\
\text { advised to facilitate } \\
\text { digestion for lactating } \\
\text { mothers. }\end{array}$ & Oral & $\begin{array}{l}\text { Oil/ Vegetables } \\
\text { (depends on the } \\
\text { cooking practice) }\end{array}$ \\
\hline \multicolumn{9}{|l|}{ Rutaceae } \\
\hline Zanthoxylum rhetsa D.C. & Chirfal & Tree & Fruit & Fever (PYR) & AR Sa 31 & $\begin{array}{l}\text { Fruits are powdered, } \\
\text { mixed with coconut oil } \\
\text { and applied at the } \\
\text { exposed part of } \\
\text { forehead. }\end{array}$ & Topical & Coconut oil \\
\hline \multicolumn{9}{|l|}{ Vitaceae } \\
\hline $\begin{array}{c}\text { Ampelocissus latifolia (Roxb.) } \\
\text { Planch. }\end{array}$ & $\begin{array}{c}\text { Draksha, } \\
\text { Jangli Draksha }\end{array}$ & Climber & Roots & $\begin{array}{c}\text { Parturition } \\
\text { complexities in } \\
\text { expectant } \\
\text { mothers } \\
\text { (GYD) }\end{array}$ & AR Sa 15 & $\begin{array}{c}\text { Oral administration of } \\
\text { root infusion to the } \\
\text { pregnant mothers prior } \\
\text { to labor, or during } \\
\text { labor. }\end{array}$ & Oral & Water \\
\hline Cissus quadrangularis L. & $\begin{array}{l}\text { Kandavela,Ha } \\
\text { dsandhi }\end{array}$ & Shrub & Stem & $\begin{array}{l}\text { Joint pain, } \\
\text { Bone fracture } \\
\text { and break } \\
(\mathrm{SKA})\end{array}$ & AR Sa 22 & $\begin{array}{l}\text { Oral administration of } \\
\text { stem infusion to the } \\
\text { fractured patients. }\end{array}$ & Oral & Water \\
\hline \multicolumn{9}{|l|}{ Xanthorrhoeaceae } \\
\hline Aloe vera (L.) Burm.f. & Koraphada & Herb & Leaves & $\begin{array}{c}\text { Fungal } \\
\text { infection at } \\
\text { Vaginal orifice } \\
\text { (GYD) }\end{array}$ & AR Sa 21 & $\begin{array}{l}\text { Juice of the bulbous } \\
\text { leaves is directly } \\
\text { applied at the female } \\
\text { genital parts. }\end{array}$ & Topical & - \\
\hline \multicolumn{9}{|l|}{ Zingiberaceae } \\
\hline Kaempferia rotunda $\mathrm{L}$. & Bhuichampa & Herb & Roots & $\begin{array}{l}\text { Pancreatic } \\
\text { disorder } \\
\text { (END) }\end{array}$ & AR Sa 09 & $\begin{array}{l}\text { Decoction is prepared } \\
\text { and drunk. }\end{array}$ & Oral & Water \\
\hline
\end{tabular}


Tab.-2: List of Plant taxa cited by tribal inhabitants of Saphale village, Palghar district of Maharashtra for preparing medicinal foods (The abbreviations associated with the ailment(s) are described in Tab.- 3) *: voucher specimen not available

\begin{tabular}{|c|c|c|c|c|c|c|c|c|c|}
\hline Family & Botanical name & $\begin{array}{l}\text { Vernacular } \\
\text { names }\end{array}$ & Habit & $\begin{array}{l}\text { Parts } \\
\text { used }\end{array}$ & $\begin{array}{l}\text { Ailments } \\
\text { treated }\end{array}$ & Vouchers & Herbal recipes & $\begin{array}{c}\text { Applications } \\
\text { to the } \\
\text { patients }\end{array}$ & $\begin{array}{c}\text { Used in a mix with } \\
\text { (solvents or other } \\
\text { plant parts) }\end{array}$ \\
\hline Pteridaceae & $\begin{array}{c}\text { Actiniopteris radiate (Sw.) } \\
\text { Link }\end{array}$ & $\begin{array}{l}\text { Mayoor } \\
\text { shikha }\end{array}$ & Fern & Leaves & $\begin{array}{c}\text { Low sperm } \\
\text { motility } \\
\text { (Oligospermia) } \\
\text { (AND) }\end{array}$ & AR Pa 3 & $\begin{array}{l}\text { Root paste is } \\
\text { administered to } \\
\text { the infertile } \\
\text { males. }\end{array}$ & Oral & $\begin{array}{l}\text { Water (depends on the } \\
\text { paste formulation) }\end{array}$ \\
\hline Acanthaceae & Adhatoda vasica Nees. & Vasaka & Herb & Leaves & $\begin{array}{c}\text { Menstrual } \\
\text { bleeding (GYD) }\end{array}$ & AR Pa 5 & $\begin{array}{l}\text { Juice of leaves } \\
\text { is consumed by } \\
\text { the women in } \\
\text { menses. }\end{array}$ & Oral & $\begin{array}{l}\text { Water (depends on the } \\
\text { juice formulation) }\end{array}$ \\
\hline Anacardiaceae & $\begin{array}{c}\text { Semecarpus anachardium } \\
\text { L.f. }\end{array}$ & Bibuwa & Tree & Seeds & Injury (BIW) & AR Pa 11 & $\begin{array}{c}\text { Seeds are } \\
\text { heated in water; } \\
\text { endosperm is } \\
\text { extracted and } \\
\text { applied at the } \\
\text { site. }\end{array}$ & Topical & Water \\
\hline Apocynaceae & Wrightia tinctoria $\mathrm{R} . \mathrm{Br}$. & $\begin{array}{l}\text { Dudhkudha, } \\
\text { Kalakudha }\end{array}$ & Tree & Stem & $\begin{array}{l}\text { Agalactorrhea, } \\
\text { (or } \\
\text { Hypogalactia) } \\
\text { (GYD) }\end{array}$ & AR Pa 15 & $\begin{array}{c}\text { Oral } \\
\text { administration of } \\
\text { stem infusion to } \\
\text { the lactating } \\
\text { mothers. }\end{array}$ & Oral & Water \\
\hline Boraginaceae & $\begin{array}{c}\text { Trichodesma indicum (L.) } \\
\text { Lehm }\end{array}$ & Chota kalpa & Herb & $\begin{array}{l}\text { Whole } \\
\text { plant }\end{array}$ & Fever (PYR) & * & $\begin{array}{l}\text { Oral ingestion of } \\
\text { plant decoction } \\
\text { is given to the } \\
\text { patients. }\end{array}$ & Oral & Water \\
\hline Celastraceae & Celastrus paniculatus Willd. & Malkangani & Climber & Roots & $\begin{array}{c}\text { Pimples and } \\
\text { blemishes (PIB) }\end{array}$ & AR Pa 2 & $\begin{array}{l}\text { Root paste is } \\
\text { applied } \\
\text { externally } \\
\text { against the } \\
\text { pimples. }\end{array}$ & Topical & $\begin{array}{l}\text { Water (depends on the } \\
\text { paste formulation) }\end{array}$ \\
\hline \multirow[t]{2}{*}{ Compositae } & Ageratum conyzoides (L.) L. & $\begin{array}{c}\text { Ghanera } \\
\text { osudi }\end{array}$ & Herb & Leaves & $\begin{array}{c}\text { Ringworm } \\
\text { infection (DED) }\end{array}$ & AR Pa 8 & $\begin{array}{l}\text { The paste of } \\
\text { leaves is mixed } \\
\text { with lime and } \\
\text { applied on the } \\
\text { rashes. }\end{array}$ & Topical & Lime \\
\hline & $\begin{array}{c}\text { Glossocardia bosvallia (L.f.) } \\
\text { DC. }\end{array}$ & $\begin{array}{l}\text { Phattar- } \\
\text { suva, Seri }\end{array}$ & Herb & $\begin{array}{l}\text { Whole } \\
\text { plant }\end{array}$ & Fever (PYR) & AR Pa 10 & $\begin{array}{l}\text { Decoction of the } \\
\text { whole plant is } \\
\text { administered to } \\
\text { the patients }\end{array}$ & Oral & Water \\
\hline Crassulaceae & $\begin{array}{l}\text { Kalanchoe pinnata (Lam.) } \\
\text { Pers. }\end{array}$ & Hadmod & Herb & Leaves & $\begin{array}{c}\text { Fracture of } \\
\text { Bones (SKA) }\end{array}$ & AR Pa 20 & $\begin{array}{l}\text { Paste of leaves } \\
\text { is applied at the } \\
\text { fracture site. }\end{array}$ & Topical & $\begin{array}{l}\text { Water (depends on the } \\
\text { paste formulation) }\end{array}$ \\
\hline
\end{tabular}




\begin{tabular}{|c|c|c|c|c|c|c|c|c|c|}
\hline Eriocaulaceae & Eriocaulom sp. & Ghasi & Herb & $\begin{array}{l}\text { Whole } \\
\text { plant }\end{array}$ & $\begin{array}{l}\text { Optical problems } \\
\text { (OPD) }\end{array}$ & AR Pa 01 & $\begin{array}{l}\text { Decoction is } \\
\text { prepared and } \\
\text { drunk. }\end{array}$ & Oral & Water \\
\hline Euphorbiaceae & Ricinus communis L. & Erand & Tree & Roots & $\begin{array}{l}\text { Dental ailments } \\
\text { (DEND) }\end{array}$ & AR Pa 04 & $\begin{array}{c}\text { Whole root is } \\
\text { chewed to treat } \\
\text { dental pain or } \\
\text { cavities. }\end{array}$ & Oral & - \\
\hline Leguminosae & $\begin{array}{c}\text { Pueraria tuberosa (Willd.) } \\
\text { DC. }\end{array}$ & $\begin{array}{l}\text { Vidarikand, } \\
\text { Ghorbel }\end{array}$ & Climber & Roots & $\begin{array}{c}\text { Abdominal/ } \\
\text { Digestive } \\
\text { disorders (GID) }\end{array}$ & AR Pa 06 & $\begin{array}{l}\text { Root decoction } \\
\text { is used to treat } \\
\text { abdominal } \\
\text { cramps and } \\
\text { stomach pain. }\end{array}$ & Oral & Water \\
\hline Loganiaceae & Strychnos potatorum L.f. & $\begin{array}{c}\text { Chilbeej, } \\
\text { Nivli }\end{array}$ & Tree & Seeds & $\begin{array}{c}\text { Stammering } \\
\text { (Speech } \\
\text { disorders) }(\mathrm{GH})\end{array}$ & * & $\begin{array}{l}\text { Decoction of ripe } \\
\text { seeds is drunk } \\
\text { by patients }\end{array}$ & Oral & Water \\
\hline Lythraceae & $\begin{array}{l}\text { Woodfordia fruticosa (L.) } \\
\text { Kurtz }\end{array}$ & Dhausad & Shrub & Flower & Dysentery (GID) & AR Pa 12 & $\begin{array}{l}\text { Dried flowers } \\
\text { are beaten up } \\
\text { with honey into } \\
\text { confection and } \\
\text { fed orally to } \\
\text { patients. }\end{array}$ & Oral & Honey \\
\hline Menispermaceae & $\begin{array}{c}\text { Cyclea peltata (Lam.) Hook. } \\
\text { F. \& Thomson }\end{array}$ & Bhanumathi & Climber & Leaves & $\begin{array}{l}\text { Gastrointestinal } \\
\text { infections of } \\
\text { infants (NPD) }\end{array}$ & AR Pa 18 & $\begin{array}{l}\text { Leaf juice is } \\
\text { given as a } \\
\text { therapy to the } \\
\text { infants. }\end{array}$ & Oral & $\begin{array}{l}\text { Water (depends on the } \\
\text { juice formulation) }\end{array}$ \\
\hline Myristicaceae & Myristica fragrans Houtt. & Jayaphala & Tree & Fruit & $\begin{array}{c}\text { Erectile } \\
\text { dysfunction } \\
\text { (AND) }\end{array}$ & AR Pa 13 & $\begin{array}{l}\text { Powder of the } \\
\text { fruits is } \\
\text { consumed with } \\
\text { water. }\end{array}$ & Oral & Water \\
\hline Rhamnaceae & Ziziphus mauritiana Lam. & Bhor, Ber & Tree & Bark & $\begin{array}{l}\text { Common Cold } \\
\text { and Cough } \\
\text { (COC) }\end{array}$ & AR Pa 14 & $\begin{array}{c}\text { Bark is } \\
\text { powdered and } \\
\text { mixed in } \\
\text { lukewarm water } \\
\text { against dry } \\
\text { cough } \\
\end{array}$ & Oral & Water \\
\hline Vitaceae & $\begin{array}{l}\text { Ampelocissus latifolia } \\
\text { (Roxb.) Baker }\end{array}$ & $\begin{array}{c}\text { Ran } \\
\text { draksha, } \\
\text { okela }\end{array}$ & Climber & Roots & $\begin{array}{l}\text { Snake bites and } \\
\text { wounds (BIW) }\end{array}$ & AR Pa 09 & $\begin{array}{l}\text { Paste of fresh } \\
\text { roots is applied } \\
\text { at the site of bite } \\
\text { and wound. }\end{array}$ & Topical & $\begin{array}{l}\text { Water (depends on the } \\
\text { paste formulation) }\end{array}$ \\
\hline Xanthorrhoeaceae & Aloe vera (L.) Burm. F. & Koraphada & Herb & Leaves & $\begin{array}{l}\text { Dental ailments } \\
\text { (DEND) }\end{array}$ & AR Pa 17 & $\begin{array}{c}\text { Oral } \\
\text { administration of } \\
\text { the juice of } \\
\text { leaves. }\end{array}$ & Oral & $\begin{array}{l}\text { Water (depends on the } \\
\text { juice formulation) }\end{array}$ \\
\hline Zingiberaceae & Zingiber cassumunar Roxb. & Pewa, Nisa & Herb & Roots & Jaundice (GD) & AR Pa 19 & $\begin{array}{l}\text { Decoction is } \\
\text { prepared and } \\
\text { drunk. }\end{array}$ & Oral & Water \\
\hline
\end{tabular}


Fidelity Level (FL)

According to Musa et al. (2011), it is an index that indicates the suitability of any plant species for the treatment of a specific illness. Friedman et al. (1986) have developed a formula for Fidelity Level as:

$$
\mathrm{FL}=\mathrm{I}_{\mathrm{p}} / \mathrm{I}_{\mathrm{u}} \times 100
$$

where $I_{p}$ shows the number of informants citing the use of plant species for a particular disease category and $l_{u}$ shows the number of informants referring to the usage of that plant species for any other disease category(s). The high value of FL reflects the worth of a particular plant species over other plants for the treatment of a specific disease- high value corresponds to the high frequency of plant usage against a particular disease. The low value of $\mathrm{FL}$ determines the use of plant species for different medicinal purposes and it confirms its low-frequency usage against a particular disease by the informants of the given study area.

\section{Relative Importance (RI)}

Bennett and Prance (2000) have proposed the following formula to calculate the index of Relative Importance (RI):

$$
R I=(P P+A C)
$$

where PP specifies the Pharmacological Properties, which are obtained by 'dividing the number of UR for plant species with the highest number of UR', while AC indicates the diseases treated related to a particular organ system of the human body. The value of $A C$ is calculated by 'dividing the number of body systems treated using a particular plant species with the highest number of disease categories treated using a most extensively used species' (Pratibhan et al. 2016). The RI value when comes 2 is the highest value possible which shall denote the highest versatility of the plant species with maximum medicinal properties (Oliveira et al. 2010).

Index of Agreement on Remedies (IAR) $\mathrm{n}$ conformity with the method adopted by Trotter and Logan (1986), Index of Agreement on Remedies (IAR) was calculated using the following formula:

$$
\operatorname{IAR}=\left(\mathrm{n}_{\mathrm{ur}}-\mathrm{n}_{\mathrm{a}}\right) /\left(\mathrm{n}_{\mathrm{ur}}-1\right)
$$

where $\mathrm{n}_{\mathrm{ur}}$ is the total number of citations registered for the species (in other words, the number of URs), and $n_{a}$ is the number of illness categories that are healed with this species. This value ranges between 0 and $1-0$ signifies the plant species which is agreed upon by the least participants whereas 1 denotes agreement of all participants upon the exclusive use of the species for the particular illness (Chellappandian et al. 2012).

Multiple Plant Use Index (MPUI): It is usually observed that an entire plant and its parts are employed for different commercial and household purposes besides the core medicinal properties. These can be a plethora of various activities like making furniture, constructing houses, feeding the cattle, culinary items, timber uses, religious aesthetics, and many more. There are no formulae for calculating the MPUI, it carried the qualitative data concerning non-medicinal uses of medicinal plants.

\section{Results and Discussion}

The present paper documented the ethnomedicinal uses of 51 medicinal plants from Ratnagiri and Palghar districts of Maharashtra. The data was assembled from 13 Hakims and 79 tribal inhabitants (39 men and 40 women) from five ethnic groups- the Dhangad tribe and the Gowari tribe from Sangameshwar Taluka, Ratnagiri district and the Marle tribe, the Gunda tribe and the Jadhav tribe from Saphale village, Palghar district.

\section{Demographic statistics of Informants}

During the survey, the investigators considered the traditional knowledge of maximum tribal inhabitants present at the given time. Certainly, there was a section of inhabitants who refrained from sharing any information primarily due to linguistic barriers and diffidence. A total of 92 tribal inhabitants belonging to different tribal groups was surveyed of which 14.13 $\%$ Hakims were all males. The highest frequency of Hakims belonged from the Gunda tribe (Palghar district) accounting for about $30.76 \%$ of the total 13 Hakims. Women informants (43.47\%) exceeded the male informants $(42.39 \%)$ by a derisory margin of $1.06 \%$. The highest female (37.5\%) and male (28.2 $\%)$ informants were recorded from the Dhangad tribe (Ratnagiri district) as depicted in Fig. 3. Quite interestingly, it was observed that females were more affable to establish conversations than malescontrary to the social notion of females being reluctant to cooperate with the male investigator(s).

On the basis of educational qualifications, two academic groups were categorized- illiterates and educated; illiterates were those who never received any kind of formal education while educated groups were further classified into subgroups of villagers who received primary education, secondary education, and senior secondary education. Only $5.42 \%$ of males out of the total count of informants were educated till secondary classes whereas females sufficed the mark of absolutely none receiving secondary education. Quite eccentric in the tribal community, there was one young girl in the 
entire study who has studied till Class- 12 which comes under the category of senior secondary education. Only $38.46 \%$ of the total count of Hakims received primary education through the formal school education system, while $46.15 \%$ of 13 Hakims informed that they never been to any academic institutions- all they knew about the medicinal plants were passed to them from their antecedents verbally. Females surpassed the males (non- Hakims) in having a higher rate of illiteracy (50.72 \%) (Fig. 4).

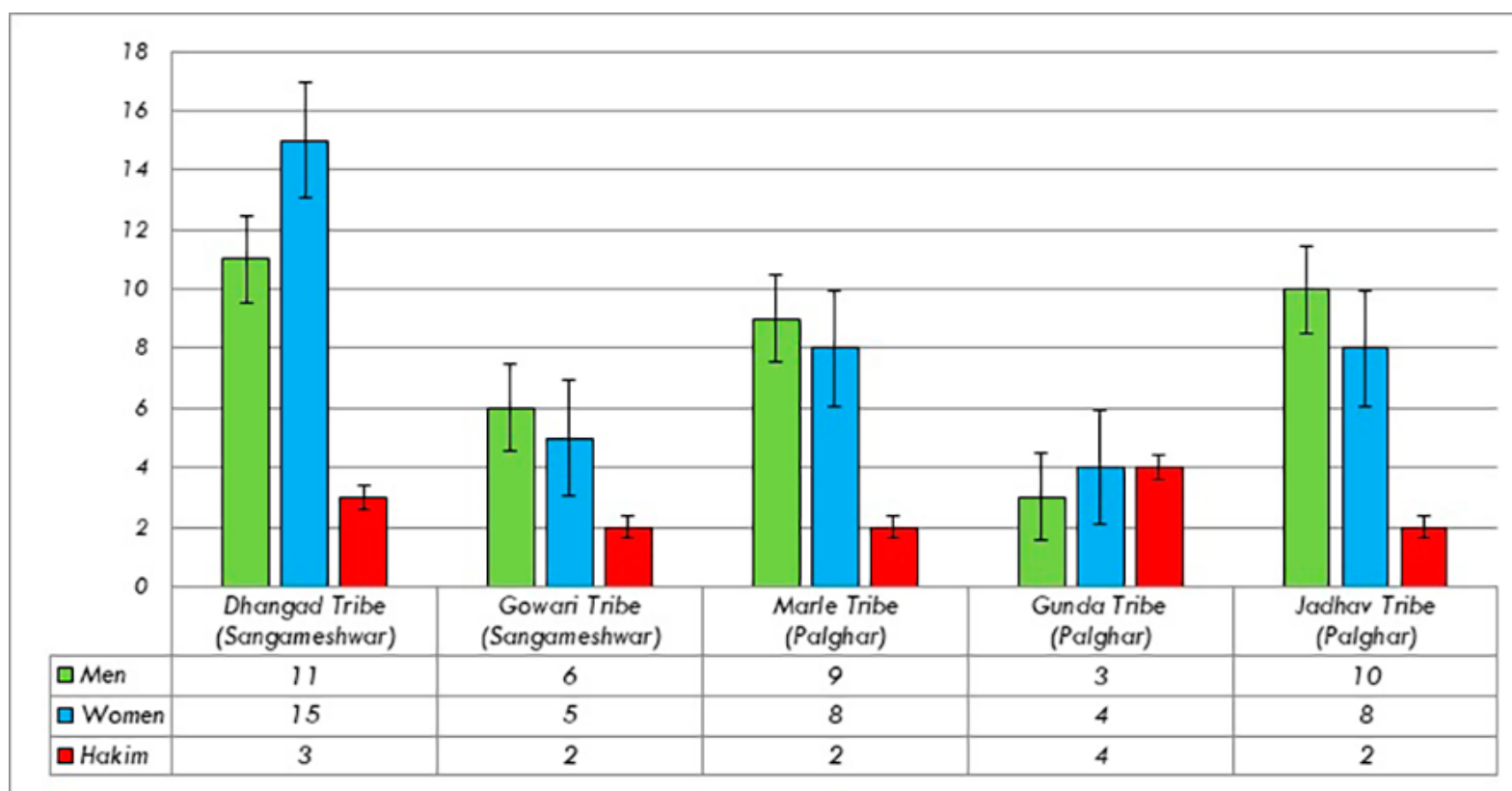

aMen $\square$ Women a Hakim

Figure 3. Distribution of tribal informants and Hakims as per ethnic groups and gender. (Vertical lines represent \pm SE)

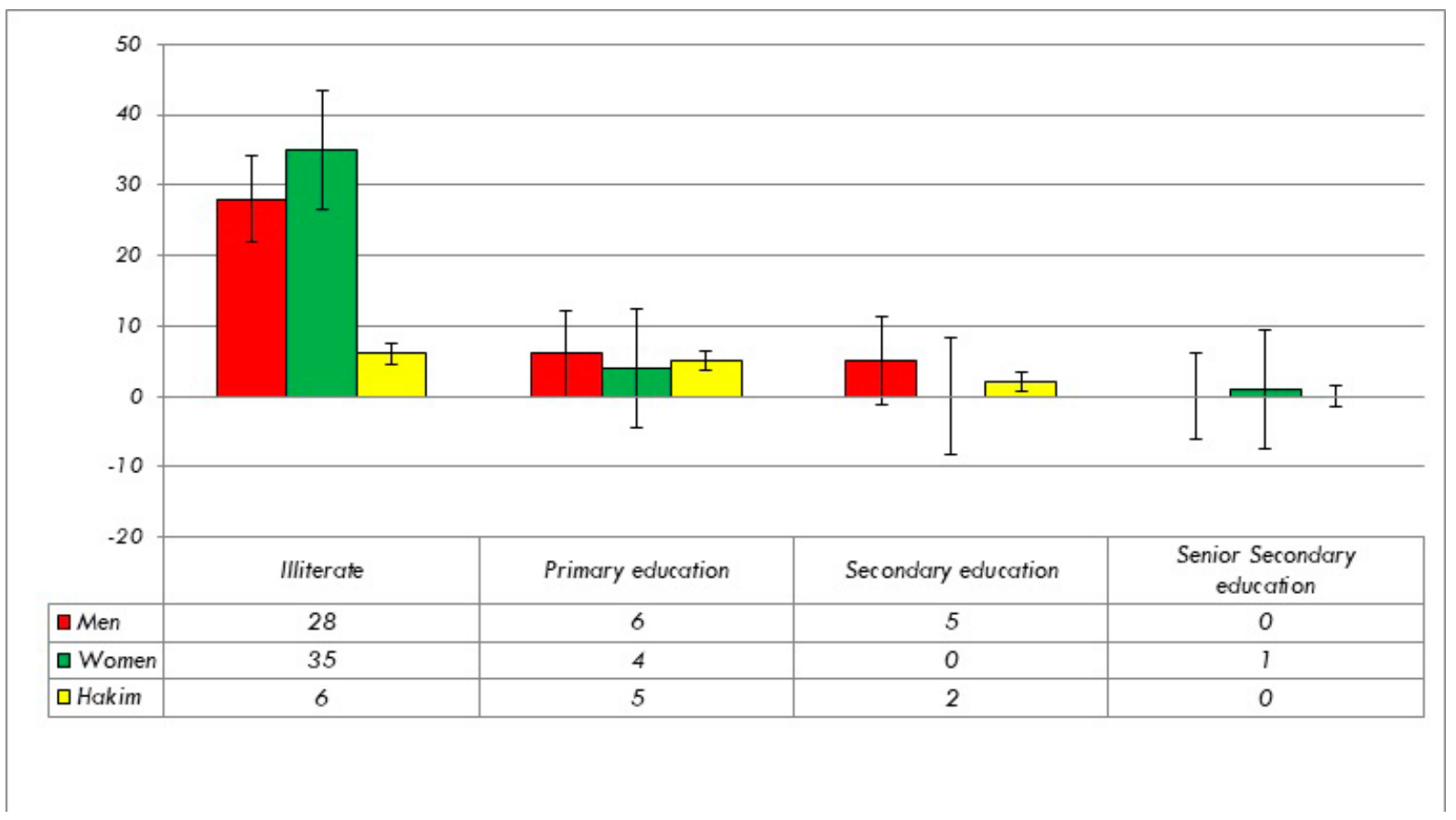

Figure 4. Distribution of tribal informants as per educational qualifications. (Vertical lines represent \pm SE) 


\section{Descriptive statistics of the TMPs}

This study documented 51 medicinal plants that were administered to the patients through different medicinal formulations. While $59 \%$ of the herbal formulations were consumed orally, $39 \%$ were applied topically for ailments like cuts, bites, wounds, and external injuries (Fig. 5). Only $2 \%$ of the herbal recipes were inhaled through smokes by prior drying of the leaves for respiratory infirmities.

The most commonly used herbal recipe was observed to be decoction (Fig. 6). This is in accordance with the past ethnobotanical researches where decoction was the major form of medicinal preparation (Ahirwar 2010, Bahmani et al. 2014, Fiscal 2017). The second most frequently used recipe was paste(s) with 9 out of 51 medicinal plants being prepared in that formulation. Juice(s), infusion(s), and fresh part(s) followed the third type with their frequency being 6 for each of them. Extract(s) and powder(s) were among the least prepared medicinal formulations with the frequency of 5 and 4 (out of 51) respectively.

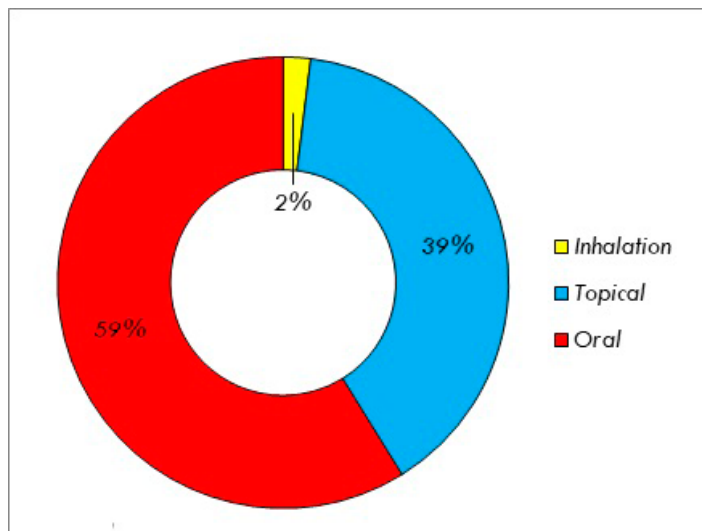

Figure 5. Mode of applications of the herbal medicinal formulations

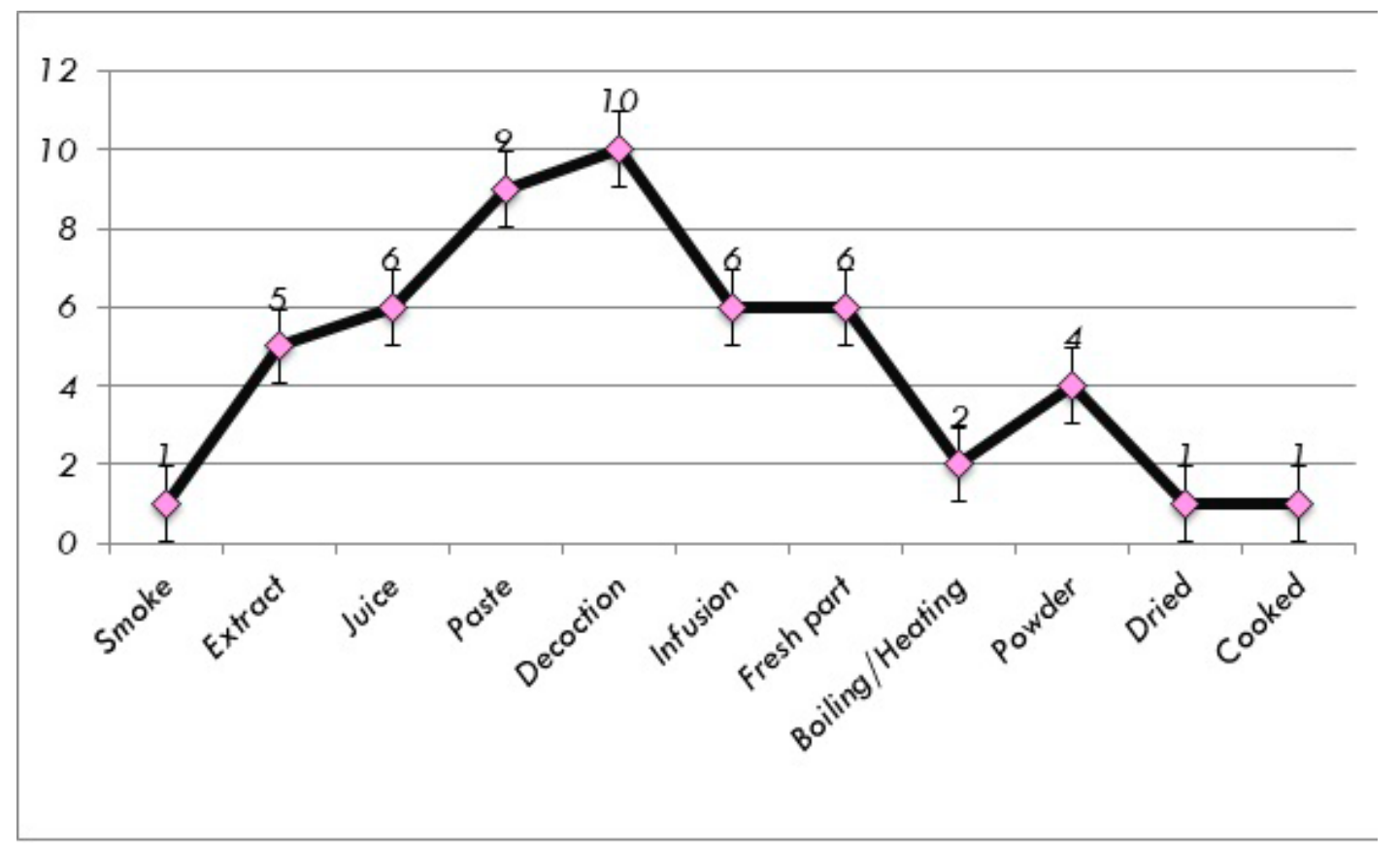

Figure 6. Mode of preparation of herbal recipes (with error bars) from the TMPs

Based on the botanical validation, the plants were classified through their life forms and life cycles. The life form patterns of the medicinal plants revealed that 20 out of 51 recorded plants were trees, followed by herbs (16; including a fern), shrubs (8), and climbers (7). The life cycles of the medicinal plants were listed as perennials, annuals, evergreen, or deciduous (Fig. 7). All their life forms were confirmed from www. flowersofindia.net and it was found that 8 out of 15 herbs (except the fern) were perennial, while all the shrubs were rendering their life cycle perennially (Fig. 8). For trees, evergreen and deciduous were the major categories which included 7 and 9 members (out of 20) respectively. The perennial climbers (7) and a deciduous fern were at the lower edge in this category (Fig. 8). Only 5 out of 15 herbs (except the ferns) lived annuallycompleting their life cycle in a single year and ensuing afresh with new germination in the successive year.

Taxonomically, the flowering plants were classified into two prime classes- Monocotyledons (or monocots) and Dicotyledons (or dicots). Furthermore, the investigators found two additional classes in the present study- Magnoliidae (specifically, subclass) and Polypodiopsida, though their representative members were lesser as compared to the other members. Dicot members exceeded the rest three classes with the highest 
frequency of $76 \%$, followed by monocots, magnoliids, and polypodiopsids with a frequency of $18 \%, 4 \%$, and $2 \%$ respectively (Fig. 9). Monocots consisted of 7 different families- maximum plants belonged to Zingiberaceae and Xanthorrhoeaceae (17 \%) as compared to magnoliids, Polypodiopsids, and other families of monocots (Fig. 10). The monocots comprised of grasses (Poaceae family) which were observed to be popular for treating ailments pertaining to the digestive system and vision. The class Polypodiopsida (division Pteridophyta) included only one member that belonged to the fern family, Pteridaceae. Piperaceae (pepper family) and Myristicaceae (nutmeg family) with a respective single-member fell under the botanical grade of Magnoliidae subclass. Dicotyledons contained 22 families with maximum members (10 \%) in the Leguminosae family (Fig. 11).

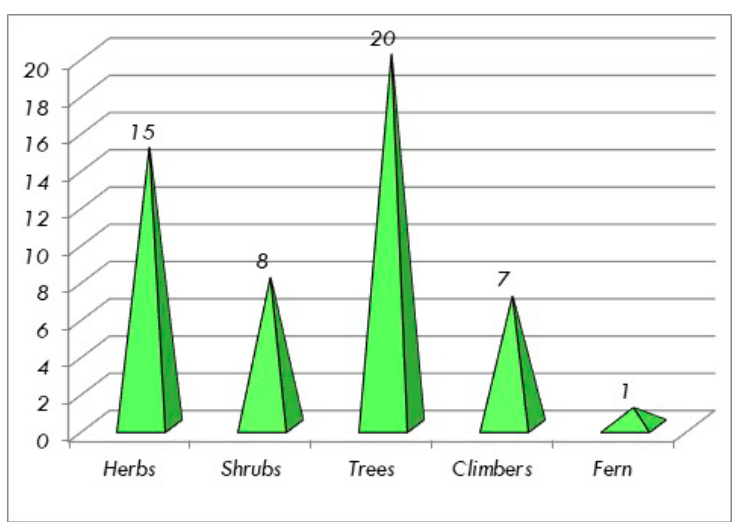

Figure 7. Distribution of the TMPs through their habits.

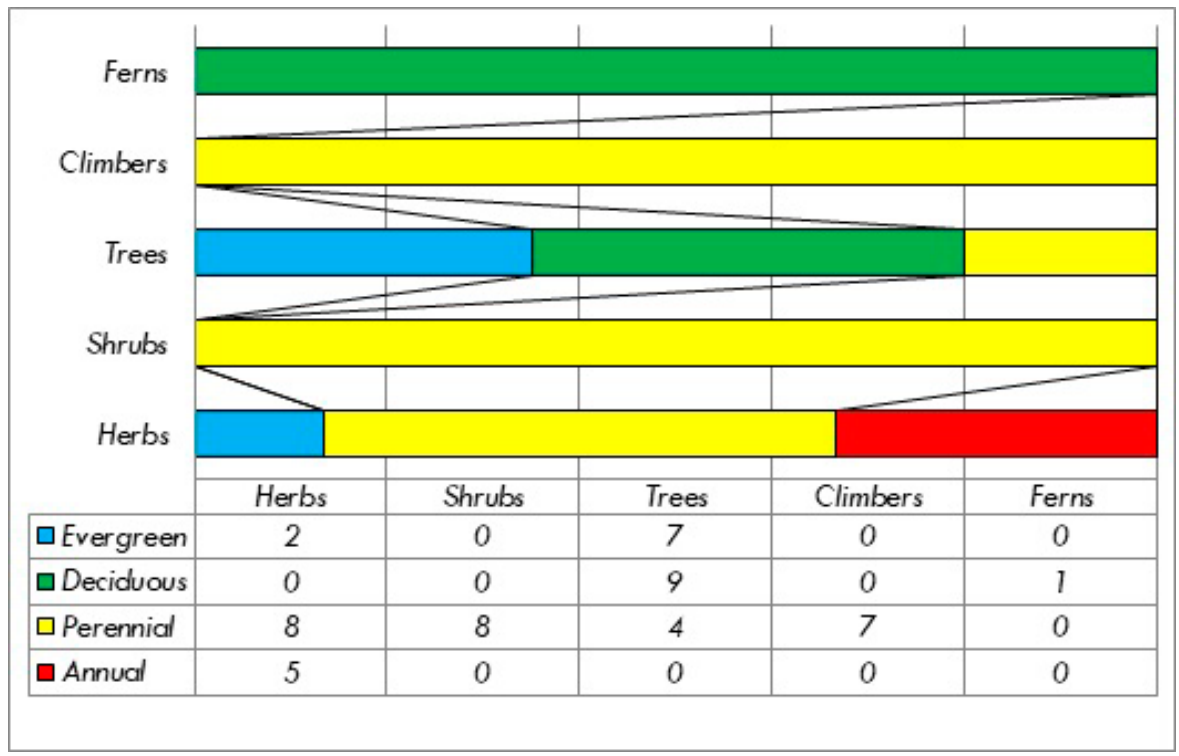

Figure 8. Distribution of the TMPs through their life cycles.

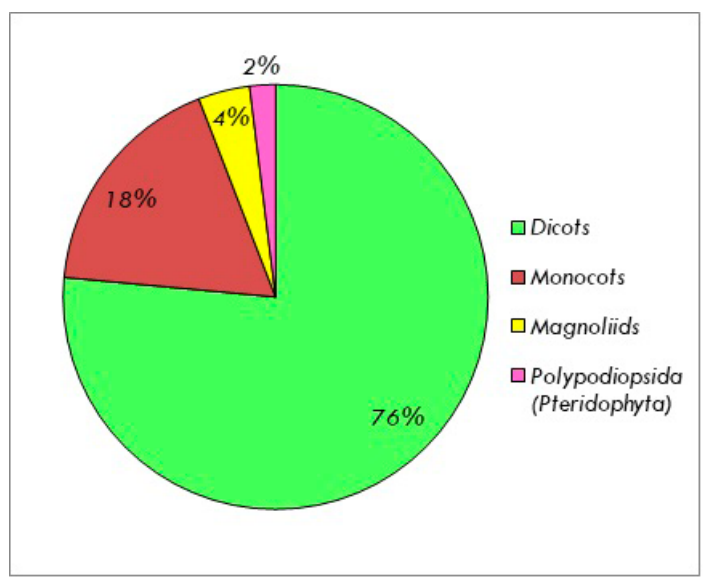

Figure 9. Distribution of the TMPs through their taxonomic classes.

Considering the objectives of the present ethnobotanical research, the medicinally important plant parts were documented through the responses of the villagers (Fig. 12). All these parts of the TMPs were signified as "therapeutically viable" by the tribal informants which were further validated by the Hakims. It was so observed that leaves (33\%) were mostly cited as the plant part with curative values, followed by roots $(19 \%)$ and bark $(16 \%)$. These results were following previous pieces of literature that found the majority of the traditional healers prescribed primarily leaves for the preparation of medicines (Amjad et al. 2015, Venkatachalapathi et al. 2018). Perhaps a plausible explanation for the high utility of leaves is that they can be easily collected with respect to the rest of the plant part(s) (Ayyanar \& Ignacimuthu, 2011). On top of that, leaves are the photosynthetically active sites and it is expected to be a potent reservoir of bioactive entities (Bahmani et al. 2014). For certain ailments, the whole plant is taken as the remedial phytotherapy 
which was about $8 \%$ of all the plant parts being used as herbal medicine. The following two tables, viz., Table 1 and Table 2, enlist the comprehensive details of the TMPs with their botanical facets and therapeutic potentialities along with their site of occurrence.

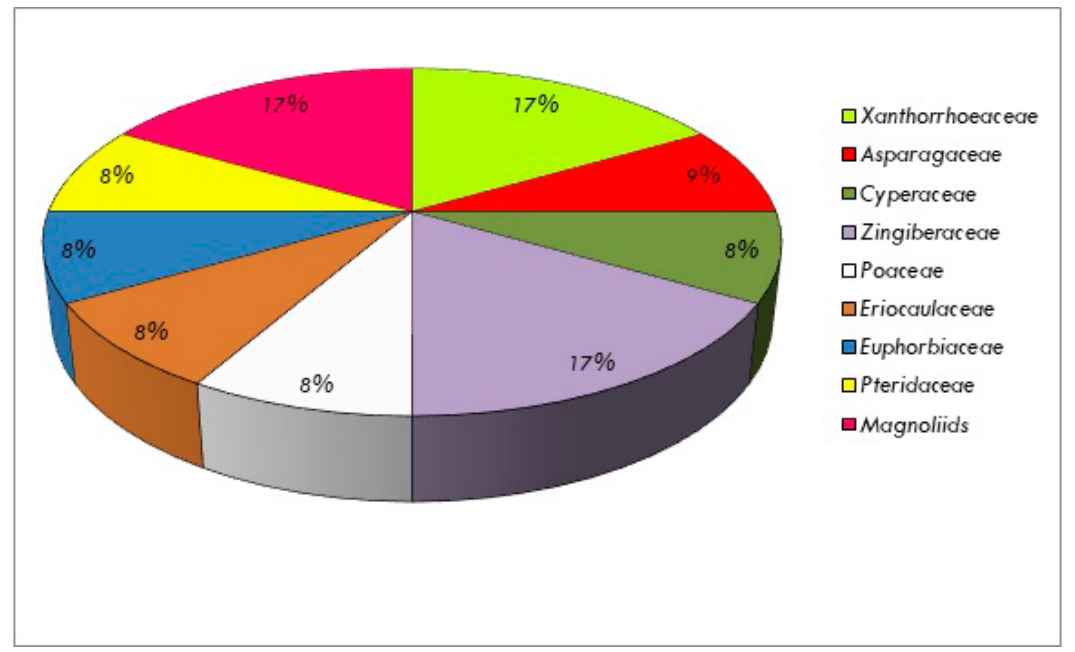

Figure 10. Frequency of the TMPs belonging to monocot, magnoliid and non-angiosperm plant families.

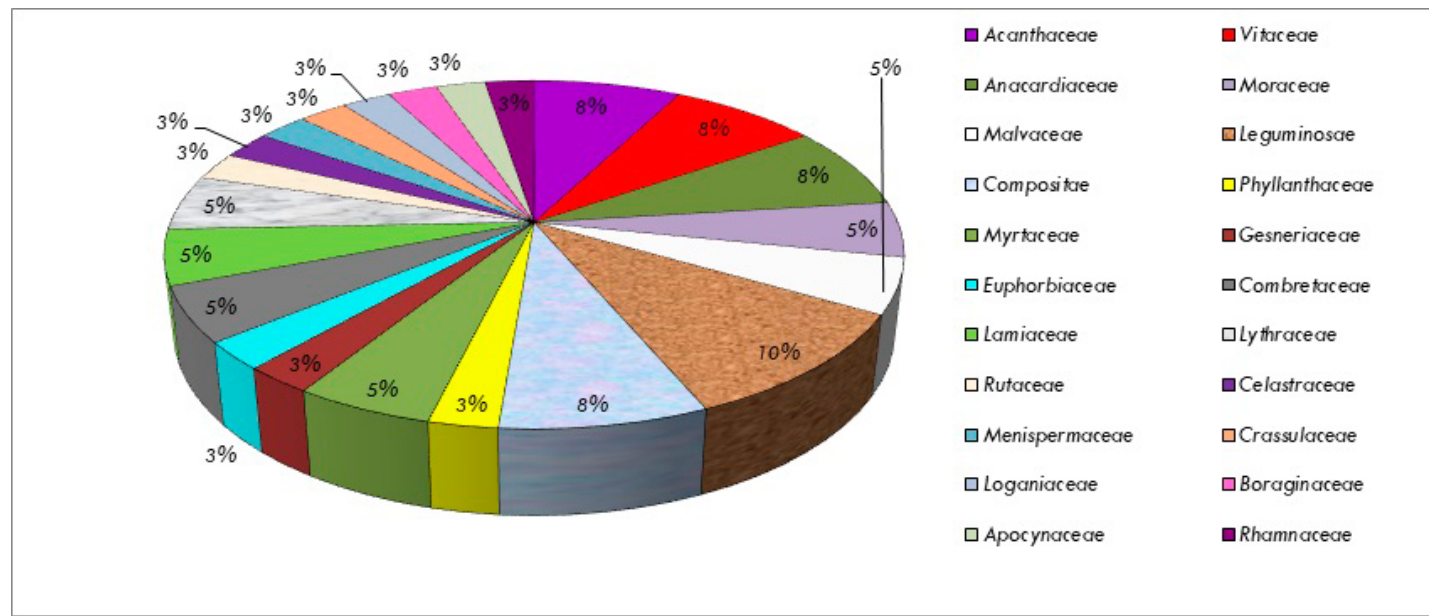

Figure 11. Frequency of the TMPs belonging to dicot families.

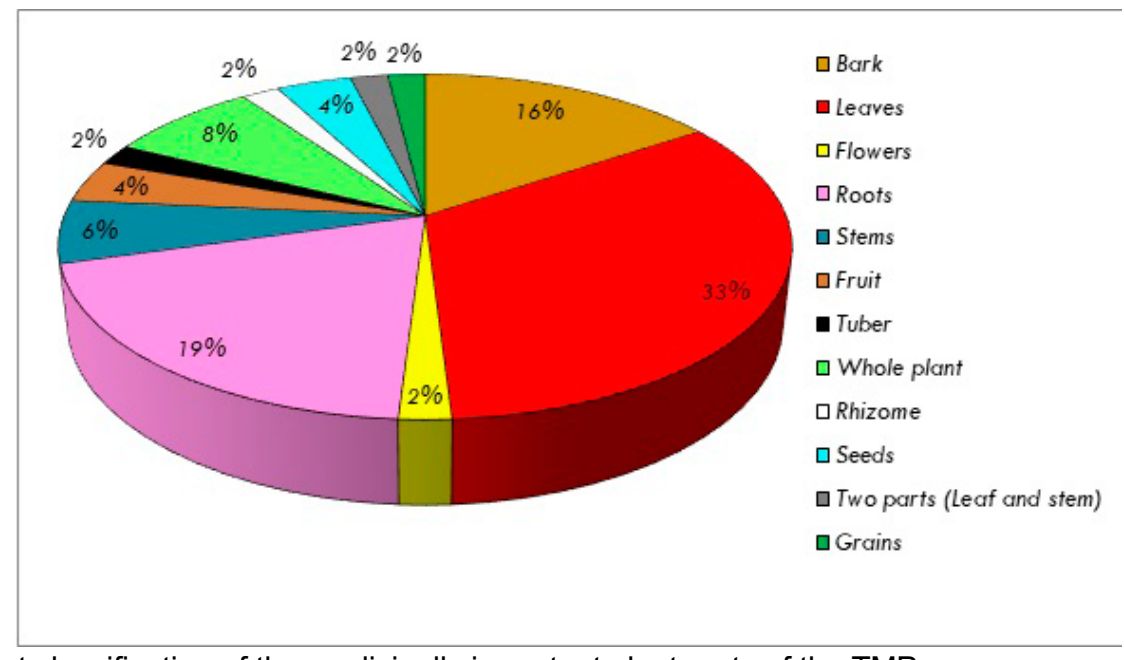

Figure 12. Percent classification of the medicinally important plant parts of the TMPs. 
Statistics of Consensus and Usage of the TMPs

$F_{\text {ic }}$ was first calculated to introspect the level of consensus among the informants and healers on the applicability of a plant for a particular ailment category. The ailment category(s) were given related medical terms and assigned an ailment code (abbreviation) as shown in Table 3. In this table, the investigators have tried to enlist the diseases based on their symptoms of certain organ system(s) of the body. The average of the entire $F_{\text {ic }}$ was counted up to 0.980 , which is indicating a quite high level of informant consensus. It can be compared with studies in Tamil Nadu by Ragupathy et al. (2008) among the "Malasars" of Velliangiri holy hills that showed average $F_{\text {ic }}$ values more than 0.70 . The following 19 major ailment categories and their $F_{i c}$ values ranged between 0.00 and 1.00 per illness category, with the highest consensus reported for as many as 7 disorders- this is conclusive evidence to stress the need for further phytopharmacological analysis to scientifically validate the species. In our study, a lower consensus factor was obtained for gynecological disorders $\left(F_{i c}=0.947\right)$ which may be logical indicative of the typical misogynistic attitude of society to discuss freely issues related to females.

Table 3: Ethnobotanical consensus index for traditional medicinal plant use categories.

\begin{tabular}{|c|c|c|c|c|}
\hline Ailment category & Ailment code & $\begin{array}{c}\text { Number of use-reports } \\
\left(\mathrm{N}_{\mathrm{Nu}}\right)\end{array}$ & Number of taxa & $\begin{array}{c}\text { Informants' Consensus } \\
\text { factor }\left(F_{i c}\right)\end{array}$ \\
\hline Andrological disorders & AND & 53 & 2 & 0.981 \\
\hline Bites, Injury and Wound & BIW & 244 & 10 & 0.963 \\
\hline Cough and cold & $\mathrm{COC}$ & 24 & 1 & 1.000 \\
\hline Dental disorders & DEND & 94 & 3 & 0.978 \\
\hline Dermatological disorders & DED & 45 & 3 & 0.955 \\
\hline Endocrinal disorders & END & 29 & 2 & 0.964 \\
\hline Gastrointestinal disorders & GID & 103 & 5 & 0.961 \\
\hline General Health & GEH & 49 & 2 & 0.979 \\
\hline Gynecological disorders & GYD & 77 & 5 & 0.947 \\
\hline Inflammatory disorders & IND & 60 & 3 & 0.966 \\
\hline Mental disorders & MED & 23 & 1 & 1.000 \\
\hline Neonatal and Paediatric disorders & NPD & 36 & 2 & 0.971 \\
\hline Optical disorders & OPD & 2 & 1 & 1.000 \\
\hline Pimples and Blemishes & PIB & 23 & 1 & 1.000 \\
\hline Pyrexia (Fever) & PYR & 112 & 4 & 0.973 \\
\hline Respiratory disorders & RED & 28 & 1 & 1.000 \\
\hline Skeleto-muscular ailments & SKA & 86 & 3 & 0.976 \\
\hline Sun stroke & SUS & 8 & 1 & 1.000 \\
\hline Veterinary disorders & VD & 20 & 1 & 1.000 \\
\hline Total & & 1116 & 51 & 0.980 \\
\hline
\end{tabular}

Among the 30 medicinal plants observed in Sangameshwar Taluka of Ratnagiri district, Terminalia paniculata had the highest UR of 33 (Table 4). The highest RFC value of 1 was observed for Terminalia paniculata, which was immediately followed by Glossocardia bosvallia with RFC value equal to 0.976. Cassia tora and Cissus quadrangularis shared the highest UV of 0.214 since they were aesthetically and therapeutically significant apart from the medicinal uses (Tab.-1). Though as many as nine plants have crossed $80 \%$ Fidelity level, Artocarpus hirsutus and Panicum miliaceum received $100 \%$ Fidelity level through the informants' responses. This fact infered that these two species were the highly espoused plants for treating the illness of a particular ailment category due to their higher healing potential. Adhatoda vasica and Aloe vera held the highest value of RI with the score of 0.944 since the informants were divided in their opinions regarding the medicinal properties and the ailment categories these plants served as a therapeutic drug. The villagers retained a certain strong point of mutual concordance in their opinions which was reflected in the IAR since four plants
Cyperus rotundus, Erythrina indica, Panicum miliaceum, and Terminalia paniculata got a score of 1 in that category.

21 plants were listed through the responses of villagers in Saphale village, Palghar district (Tab.-5) where Ampelocissus latifolia got the highest UR of 39. Aloe vera received the highest RFC of 1 with most of the Palghar inhabitants endorsing Aloe as an assertive medicinal herb. The highest UV of 0.16 belonged to Trichodesma indicum with a multitude of uses as per the villagers. Adhatoda vasica has 100 $\%$ Fidelity level (FL) in Saphale village which showed its acceptance as a medicinal herb for a particular ailment category (Table 5). The highest score of RI was accorded to Celastrus paniculatus (1.725) for its vivid pharmaceutical properties. Cyclea peltata was recognized as a medicinal herb by the folks collaboratively with the highest IAR of 1 while Eriocaulom sp. was the debated plant $(I A R=0)$ over its credibility as a medicinal herb for the particular ailment category. 
Table 4: Mathematical interpretation of medicinal efficacies of TMPs from Sangameshwar Taluka, Ratnagiri district

\begin{tabular}{|c|c|c|c|c|c|c|c|}
\hline BOTANICAL NAME & $\begin{array}{l}\text { USE } \\
\text { REPORTS } \\
\text { (UR) }\end{array}$ & $\begin{array}{l}\text { RELATIVE } \\
\text { FREQUENCY } \\
\text { CITATION } \\
\text { (RFC) }\end{array}$ & $\begin{array}{l}\text { USE } \\
\text { VALUE } \\
\text { (UV) }\end{array}$ & $\begin{array}{l}\text { FIDELITY } \\
\text { LEVEL (FL) }\end{array}$ & $\begin{array}{l}\text { RELATIVE } \\
\text { IMPORTANCE } \\
\text { (RI) }\end{array}$ & $\begin{array}{l}\text { INDEX OF } \\
\text { AGREEMENT } \\
\text { ON REMEDIES } \\
\text { (IAR) }\end{array}$ & $\begin{array}{l}\text { MULTIPLE PART USE } \\
\text { INDEX (MPUI) }\end{array}$ \\
\hline Adhatoda vasica Nees & 28 & 0.881 & 0.167 & 64.286 & 0.944 & 0.926 & - \\
\hline Aloe vera (L.) Burm.f. & 19 & 0.738 & 0.143 & 63.158 & 0.944 & 0.778 & $\begin{array}{c}\text { Ornamental plant for small } \\
\text { plots. }\end{array}$ \\
\hline Ampelocissus latifolia (Roxb.) Planch. & 8 & 0.667 & 0.071 & 87.5 & 0.445 & 0.857 & - \\
\hline Anacardium occidentale L. & 7 & 0.810 & 0.095 & 85.714 & 0.611 & 0.667 & $\begin{array}{l}\text { Edible nut meant for culinary } \\
\text { dishes. }\end{array}$ \\
\hline Artocarpus hirsutus Lam. & 20 & 0.667 & 0.119 & 100 & 0.667 & 0.947 & $\begin{array}{l}\text { Plant part(s) are used for } \\
\text { edible dishes. }\end{array}$ \\
\hline Asteracantha longifolia Nees & 18 & 0.905 & 0.071 & 44.4 & 0.445 & 0.941 & - \\
\hline Cassia tora L. & 30 & 0.714 & 0.214 & 50 & 1.5 & 0.724 & $\begin{array}{c}\text { Plant is used as a natural } \\
\text { pesticide. }\end{array}$ \\
\hline Ceiba pentandra (L.) Gaertn. & 11 & 0.548 & 0.071 & 81.818 & 0.5 & 0.8 & $\begin{array}{c}\text { Birds nest in it, and mammals } \\
\text { use the huge branches as } \\
\text { highways. }\end{array}$ \\
\hline $\begin{array}{c}\text { Chlorophytum tuberosum (Roxb.) } \\
\text { Baker }\end{array}$ & 23 & 0.667 & 0.048 & 34.783 & 0.389 & 0.909 & $\begin{array}{l}\text { 1. Bulbs and leaves are } \\
\text { edible. } \\
\text { 2. Bulbs and leaves are } \\
\text { pounded into flour for Bread. }\end{array}$ \\
\hline Cissus quadrangularis $\mathrm{L}$. & 31 & 0.952 & 0.214 & 70.968 & 1.5 & 0.733 & - \\
\hline Cyperus rotundus $\mathrm{L}$. & 8 & 0.476 & 0.119 & 37.500 & 0.611 & 1 & - \\
\hline Dolichos trilobus L. & 11 & 0.738 & 0.048 & 81.818 & 0.333 & 0.9 & Culinary purpose(s). \\
\hline Erythrina indica Lam. & 13 & 0.690 & 0.071 & 38.462 & 0.389 & 1 & $\begin{array}{c}\text { Flowers are meant for divine } \\
\text { veneration. }\end{array}$ \\
\hline Ficus racemosa $\mathrm{L}$. & 16 & 0.857 & 0.071 & 43.750 & 0.444 & 0.933 & Children play with the figs. \\
\hline Glossocardia bosvallia (L.f.) DC. & 27 & 0.976 & 0.143 & 74.074 & 0.833 & 0.923 & $\begin{array}{l}\text { Edible bulbs and leaves are } \\
\text { meant for culinary purposes. }\end{array}$ \\
\hline Kaempferia rotunda L. & 18 & 0.952 & 0.143 & 77.778 & 0.778 & 0.941 & $\begin{array}{c}\text { Flowers have aesthetic } \\
\text { value(s). }\end{array}$ \\
\hline Mangifera indica L. & 25 & 0.786 & 0.071 & 76 & 0.5 & 0.917 & $\begin{array}{l}\text { Fruits are edible and leaves } \\
\text { are addressed for many } \\
\text { devotional activities. }\end{array}$ \\
\hline Panicum miliaceum L. & 9 & 0.619 & 0.048 & 100 & 0.278 & 1 & Crops are edible. \\
\hline Phyllanthus emblica L. & 6 & 0.524 & 0.095 & 50 & 0.556 & 0.8 & $\begin{array}{c}\text { Fruits are edible (rich in } \\
\text { Vitamin-C). }\end{array}$ \\
\hline Piper betle L. & 18 & 0.881 & 0.167 & 83.3 & 1.333 & 0.471 & $\begin{array}{c}\text { Leaves are edible (usually as } \\
\text { dessert). }\end{array}$ \\
\hline
\end{tabular}




\begin{tabular}{|c|c|c|c|c|c|c|c|}
\hline Psidium guajava L. & 27 & 0.905 & 0.190 & 85.185 & 1 & 0.962 & Fruits are edible. \\
\hline Rhynchoglossum obliquum Blume & 32 & 0.833 & 0.071 & 84.375 & 0.5 & 0.935 & - \\
\hline Ricinus communis $\mathrm{L}$. & 25 & 0.738 & 0.167 & 72 & 0.889 & 0.958 & - \\
\hline Syzigium cumini (L.) Skeels & 19 & 0.619 & 0.119 & 78.947 & 0.667 & 0.944 & Fruits are edible. \\
\hline Terminalia bellerica (Gaertn.) Roxb. & 21 & 0.548 & 0.048 & 76.19 & 0.333 & 0.95 & Dried kernels are smoked. \\
\hline Terminalia paniculata Roth. & 33 & 1 & 0.119 & 90.909 & 0.611 & 1 & $\begin{array}{l}\text { 1. Fruits are used for tanning } \\
\text { \& dyeing. } \\
\text { 2. The tree is extensively } \\
\text { utilized in pharmaceutical, } \\
\text { timber tannin, leather and silk } \\
\text { industries. }\end{array}$ \\
\hline Urena lobata L. & 26 & 0.810 & 0.167 & 73.077 & 0.944 & 0.92 & $\begin{array}{l}\text { Attractive flower(s) for } \\
\text { pollinator(s). }\end{array}$ \\
\hline Vitex negundo L. & 21 & 0.690 & 0.071 & 66.667 & 0.778 & 0.65 & $\begin{array}{c}\text { The leaves are used as a } \\
\text { mosquito repellent. }\end{array}$ \\
\hline Vitex trifolia L. & 22 & 0.881 & 0.119 & 90.909 & 0.722 & 0.905 & $\begin{array}{l}\text { Wood is used for constructing } \\
\text { light furniture(s). }\end{array}$ \\
\hline Woodfordia fruticosa (L.) Kurz. & 28 & 0.762 & 0.095 & 32.143 & 0.556 & 0.963 & - \\
\hline Zanthoxylum rhetsa D.C. & 18 & 0.619 & 0.190 & 42.857 & 1.889 & 0 & $\begin{array}{l}\text { Aromatic stem and fruit used } \\
\text { to make perfume(s). }\end{array}$ \\
\hline
\end{tabular}


Table 5: Mathematical interpretation of medicinal efficacies of TMPs from Saphale village, Palghar district

\begin{tabular}{|c|c|c|c|c|c|c|c|}
\hline BOTANICAL NAME & $\begin{array}{l}\text { USE } \\
\text { REPORTS } \\
\text { (UR) }\end{array}$ & $\begin{array}{c}\text { RELATIVE } \\
\text { FREQUENCY } \\
\text { CITATION } \\
\text { (RFC) } \\
\end{array}$ & $\begin{array}{l}\text { USE } \\
\text { VALUE } \\
\text { (UV) }\end{array}$ & $\begin{array}{l}\text { FIDELITY } \\
\text { LEVEL (FL) }\end{array}$ & $\begin{array}{l}\text { RELATIVE } \\
\text { IMPORTANCE } \\
\text { (RI) }\end{array}$ & $\begin{array}{c}\text { INDEX OF } \\
\text { AGREEMENT } \\
\text { ON REMEDIES } \\
\text { (IAR) } \\
\end{array}$ & $\begin{array}{l}\text { MULTIPLE PART } \\
\text { USE INDEX(MPUI) }\end{array}$ \\
\hline Actiniopteris radiate (Sw.) Link & 20 & 0.76 & 0.04 & 65 & 0.45 & 0.842 & $\begin{array}{c}\text { Ornamental plant- } \\
\text { leaves are decorative. }\end{array}$ \\
\hline Adhatoda vasica Nees. & 17 & 0.78 & 0.08 & 100 & 0.65 & 0.875 & - \\
\hline Ageratum conyzoides (L.) L. & 19 & 0.62 & 0.06 & 47.368 & 0.525 & 0.889 & $\begin{array}{l}\text { Leaves when burnt ac } \\
\text { as insect repellents. }\end{array}$ \\
\hline Aloe vera (L.) Burm. F. & 35 & 1 & 0.06 & 28.571 & 0.625 & 0.882 & $\begin{array}{c}\text { Ornamental plant for } \\
\text { small plots. }\end{array}$ \\
\hline Ampelocissus latifolia (Roxb.) Baker & 39 & 0.84 & 0.04 & 33.333 & 0.35 & 0.97 & - \\
\hline Celastrus paniculatus Willd. & 23 & 0.26 & 0.14 & 76.923 & 1.725 & 0.273 & - \\
\hline $\begin{array}{c}\text { Cyclea peltata (Lam.) Hook. F. \& } \\
\text { Thomson }\end{array}$ & 15 & 0.5 & 0.02 & 46.667 & 0.175 & 1 & - \\
\hline Eriocaulom sp. & 2 & 0.68 & 0.12 & 50 & 0.85 & 0 & - \\
\hline Glossocardia bosvallia (L.f.) DC. & 35 & 0.78 & 0.10 & 34.286 & 0.775 & 0.942 & - \\
\hline Kalanchoe pinnata (Lam.) Pers. & 29 & 0.8 & 0.04 & 82.759 & 0.35 & 0.964 & $\begin{array}{l}\text { Scientific aid to teach } \\
\text { vegetative propagation } \\
\text { to science students. }\end{array}$ \\
\hline Myristica fragrans Houtt. & 33 & 0.92 & 0.06 & 69.697 & 0.525 & 0.938 & $\begin{array}{l}\text { Seeds are used to } \\
\text { enhance food's flavor. }\end{array}$ \\
\hline Pueraria tuberosa (Willd.) DC. & 19 & 0.7 & 0.02 & 42.105 & 0.575 & 0.556 & - \\
\hline Ricinus communis L. & 32 & 0.92 & 0.04 & 46.875 & 0.35 & 0.968 & - \\
\hline Semecarpus anachardium L.f. & 28 & 0.74 & 0.06 & 28.571 & 1.025 & 0.556 & $\begin{array}{l}\text { 1. Kernels are eaten. } \\
\text { 2. Nut imparts color } \\
\text { which is used as dye. }\end{array}$ \\
\hline Strychnos potatorum L.f. & 33 & 0.84 & 0.06 & 15.152 & 1.325 & 0.438 & $\begin{array}{l}\text { 1. Seeds are used to } \\
\text { cleanse fowl water. } \\
\text { 2. Gums (from seeds) } \\
\text { used in Paper/Textile } \\
\text { industries. }\end{array}$ \\
\hline Trichodesma indicum (L.) Lehm & 34 & 0.9 & 0.16 & 61.765 & 2 & 0.424 & Culinary purposes. \\
\hline Woodfordia fruticosa (L.) Kurtz & 31 & 0.9 & 0.04 & 25.806 & 0.35 & 0.967 & - \\
\hline Wrightia tinctoria R. Br. & 22 & 0.76 & 0.08 & 77.273 & 0.85 & 0.714 & $\begin{array}{l}\text { 1. Wood is strong } \\
\text { enough to make cups, } \\
\text { toys, etc. } \\
\text { 2. A few drops of its } \\
\text { sap in milk prevent } \\
\text { curdling. }\end{array}$ \\
\hline Zingiber cassumunar Roxb. & 26 & 0.88 & 0.12 & 61.538 & 1.15 & 0.72 & $\begin{array}{l}\text { Pieces of the herb are } \\
\text { used as flavoring } \\
\text { agents in dishes. }\end{array}$ \\
\hline Ziziphus mauritiana Lam. & 24 & 0.96 & 0.06 & 12.500 & 0.475 & 0.957 & - \\
\hline
\end{tabular}


MPUI does not bear any scores, it drafted all the (qualitative) non-medicinal uses of the concerned TMPs that included day-to-day uses of the plant(s) and their corresponding parts.

\section{Conclusions}

The 51 documented plant species indicated the strong traditional knowledge of the tribal villagers on medicinal plants in both the study sites. One of the striking observations included that the same plants were known by different vernacular names and were reported to treat dissimilar ailments in the two study areas. The six plants- Adhatoda vasica, Aloe vera, Ampelocissus latifolia, Glossocardia bosvallia, Ricinus communis, and Woodfordia fruticose- were the common TMPs in both the study areas. Both the study areas are geographically quite far (approx. 440 $\mathrm{km}$ ) from each other inhabiting tribal from different genealogical backgrounds, therefore, there is no point of clash of consensus between their opinions. The change in the vernacular names may be attributed to ethnicity and migration of inhabitants, while the ailment categories are completely a matter of opinion influenced by the diseases with a higher frequency of occurrence at the concerned site(s). Therefore, in addition to demographic details, the prevalence of ailment types must be considered in ethnobotanical studies such that the retrieved information can be further employed to yield puissant drugs.

In the scope of the present study, the informant consensus level is 0.980 , which can be considered as an outcome of reliable data. Based on consensus, this study shall serve as a paving stone for the commencement of many such ethnobotanical studies in Maharashtra and other parts of our nation. The TMPs with high important values ( $\left.F_{i c}, U V, F L\right)$ are highly recommended for further pharmacological tests.

\section{Declarations}

Abbreviations: TMPs- Traditional medicinal plants Ethics approval and consent to participate: The research team explained to the elders and the local community members the purpose of the study before the data collection. The participants were asked to sign an informed consent form, as instructed by the Ethical and Scientific Committee of K.J. Somaiya College of Science and Commerce, Mumbai and University of Mumbai, Mumbai, India.

Consent for publication: Not applicable.

Availability of data and materials: The data was not deposited in public repositories.

Competing interests: The authors declare no competing interests. There is not any clash of attentiveness in this study.
Funding: This research did not receive any specific grant from funding agencies in the public, commercial, or not-for-profit sectors.

Authors' contributions: AR carried out fieldwork, developed the questionnaire, data analysis, and drafted the manuscript. AR and SJ conceptualized the study and designed the methods. The work was supervised by SJ. Both authors read, reviewed and approved the final version of the manuscript.

\section{Acknowledgements}

The authors thank Dr. Pradnya Prabhu, Principal, KJSSC Mumbai for permitting the study. We are also grateful to the tribal informants for sharing their knowledge. We express gratitude to Dr. Suchendra Dutta, Asst. Professor, R. D. National College, Bandra (W), Mumbai for guiding in the taxonomic classification of the plant species. We are extremely obliged to Mr. Deependu Das, Mr. Ganesh Bhosale, Mr. Neeraj Bhise and Mr. Ravindra Sitaram Shetye for providing on-field scientific, vernacular, and health support.

\section{Literature cited}

Abu-Irmaileh BE, Afifi FU. 2003. Herbal medicine in Jordan with special emphasis on commonly used herbs. Journal of Ethnopharmacology 89(2-3):193197.

Ahirwar RK. 2010. Ethnomedicinal uses of plant roots from Shadol district of M.P. India. Indian Journal of Applied and Pure Biology 25(1):71-76.

Amjad MS, Arshad M, Qureshi R. 2015. Ethnobotanical inventory and folk uses of indigenous plants from Pir Nasoora National Park, Azad Jammu and Kashmir. Asian Pacific Journal of Tropical Biomedicine 5(3):234-241.

Arora RK. 1997. Ethnobotany and its role in the conservation and use of Plant Genetic Resources in India. Ethnobotany 9: 6-15.

Ayyanar M, Ignacimuthu S. 2011. Ethnobotanical survey of medicinal plants commonly used by Kani tribals in Tirunelveli hills of Western Ghats, India. Journal of Ethnopharmacology 134(3):851-864.

Azaizeh H, Fulder S, Khalil K, Said O. 2003. EthnoMedicinal Knowledge of Local Area Practitioners in the Middle East Region. Fitoterapia 14:98-108.

Bahmani M, Zargaran A, Rafieian-Kopaei M, Saki K. 2014. Ethnobotanical study of medicinal plants used in the management of diabetes mellitus in the Urmia, Northwest Iran. Asian Pacific Journal of Tropical Medicine 7 (Suppl. 1):S348-S354.

Bennett BC, Prance GT. 2000. Introduced plants in the indigenous pharmacopeia of Northern South America. Economic Botany 54(1):90-102. 
Bhat JA, Kumar M, Negi AK, Todaria NP. 2013. Informants consensus on ethnomedicinal plants in Kedarnath Wildlife Sanctuary of Indian Himalayas. Journal of Medicinal Plants Research 7 (4):148-154.

Brown CH. 1977. Folk Botanical Life-Forms: Their Universality and Growth. American Anthropologist 79:317-342.

Budha-Magar S, Bhandari P, Ghimire SK. 2020. Ethno-medicinal survey of plants used by Magar (Kham) community, Rolpa district, Western Nepal. Ethnobotany Research and Applications 19:1-29.

Canales M, Herńandez T, Caballero J, Romo de Vivar A, Avila G, Duran A, Lira R. 2005. Informant consensus factor and antibacterial activity of the medicinal plants used by the people of San Rafael Coxcatlan, Puebla, Mexico. Journal of Ethnopharmacology 97:429-439.

Cavéchia LA, Proença CEB. 2007. Cultural rescue of uses of native Cerrado plants by the traditional population of the region of the current Federal District. Heringeriana 1(2):11-24.

Chakraborty MK, Bhattacharjee A. 2006. Some common ethnomedicinal uses of various diseases in Purulia district. West Bengal. Indian Journal of Traditional Knowledge 5(4):554-558.

Chandra K. 1995. An Ethnobotanical study on some medicinal plants of district Palamau (Bihar) Sachitra Ayurveda (Aug.):311-314.

Chellappandian M, Mutheeswaran S, Pandikumar P, Duraipandiyan V, Ignacimuthu S. 2012. Quantitative ethnobotany of traditional Siddha medical practitioners from Radhapuram taluk of Tirunelveli District, Tamil Nadu, India. Journal of Ethnopharmacology 143:540-547.

Das PK, Mondal A.K. 2013. Ethno-medicinal plants used as phytotherapeutic agents for curing various wounds in the hilly region of Paschim Medinipur district, West Bengal, India. In Ethnobotanical Studies in India. Edited by Sanjeev Kumar. Deep Publications, Kolkata, India, Pp. 211-219.

Dileep P, Geetha GN. 2015. Taxonomic and Ethnobotanical studies of Grasses used by Tribals of Wayanad District, Kerala, South Western Ghats of India. Journal of Global Sciences 4(5):2212-2235.

Esakkimuthu S, Darvin SS, Mutheeswaran S, Paulraj MG, Pandikumar P, Ignacimuthu S, Al-Dhabi NA. 2018. A study on food-medicine continuum among the non-institutionally trained Siddha practitioners of Tiruvallur district, Tamil Nadu, India. Journal of Ethnobiology and Ethnomedicine 14(45):1-29.

Fiscal RR. 2017. Ethnomedicinal plants used by traditional healers in Laguna, Philippines. Asia Pacific Journal of Multidisciplinary Research 5(4):132-137.
Friedman J, Yaniv Z, Dafni A, Palewitch D. 1986. A preliminary classification of the healing potential of medicinal plants, based on a rational analysis of an ethnopharmacological field survey among Bedouins in the Negev Desert, Israel. Journal of Ethnopharmacology 16(2-3):275-287.

Gazzaneo LRS, de Lucena RFP, de Albuquerque UP. 2005. Knowledge and use of medicinal plants by local specialists in a region of Atlantic Forest in the state of Pernambuco (Northeastern Brazil). Journal of Ethnobiology and Ethnomedicine 1(9):1-8.

Government of India Census. 2011. Primary Census Abstracts, Registrar General of India, Ministry of Home Affairs, Government of India. Available at: http://www.censusindia.gov.

Gupta DK, Gupta G. 2017. Diversity of Ethno Medician Plant in Dist. Balod (C.G). IOSR Journal of Pharmacy and Biological Sciences 12(3):80-89.

Heinrich M, Ankli A, Frei B, Weimann C, Sticher O. 1998. Medicinal plants in Mexico: Healers' consensus and cultural importance. Social Science \& Medicine 47(11):1859-1871.

Heinrich M, Edwards S, Moerman DE, Leonti M. 2009. Ethnopharmacological field studies: a critical assessment of their conceptual basis and methods. Journal of Ethnopharmacology 124(1):1-17.

Islam MA, Jha RK. 2003. Ethnopharmacology of tribals of Ranchi district of Jharkhand. Journal of Economic and Taxonomic Botany 27(2):300-310.

Jain SP. 1996. Ethno- Medico - Botanical survey of Chaibasa singbhum district, Bihar. Journal of Economic and Taxonomic Botany Addl Series 12:403-407.

Jayasundar R. 2010. Ayurveda: a distinctive approach to health and disease. Current Science 98(7):908-914.

Jones VH. 1941. The nature and scope of ethnobotany. Chronica Botanica 6:219-221.

Joshi K \& Joshi AR. 2008. Ethnobotanical studies on some lower plants of the central development region, Nepal. Ethnobotanical Leaflets 12:832-40.

Katewa SS, Chaudhary BL, Jain A. 2004. Folk herbal medicines from tribal area of Rajasthan, India. Journal of Ethnopharmacology 92:41-46.

Kayne SB. 2010. Traditional medicine: a global perspective. Pharmaceutical Press, London, U.K.

Kloutsos G, Balatsouras DG, Kaberos AC, Kandiloros D, Ferekidis E, Economou C. 2001. Upper airway edema resulting from use of Ecballium elaterium. Laryngoscope 11 (9):1652-1655.

Kuvar SD, Bapat UC. 2010. Medicinal plants used by Kokani tribals of Nasik district Maharashtra to cure cuts and wounds. Indian Journal of Traditional Knowledge 9(1):114-115. 
Mabogo DEN. 2012. The ethnobotany of the Vhavenda. Masters dissertation. University of Pretoria, South Africa.

McMillen H. 2012. Ethnobotanical knowledge transmission and evolution: the case of medicinal markets in Tanga, Tanzania. Economic Botany 66(2):121-131.

Ministério da S. 2005. Produzir e aplicar conhecimento na busca da universalidade e equidade, com qualidade da assistência à saúde da população. Secretaria de Ciência, Tecnologia e Insumos Estratégicos. Anais da 2a. Conferência Nacional de Ciência, Tecnologia e Inovação em Saúde. SCTIE/DECIT, CNS. Brasilia.

Mitra S, Mukherjee SK. 2005. Ethnobotanical usages of grasses by the tribals of West Dinajpur district, West Bengal. Indian Journal of Traditional Knowledge 4(4):396-402

Murtem G, Chaudhry P. 2016. An Ethnobotanical Study of Medicinal Plants Used by the Tribes in Upper Subansiri District of Arunachal Pradesh, India. American Journal of Ethnomedicine 3(3):35-49.

Musa MS, Abdelrasool FE, Elsheikh EA, Ahmed LAMN, Mahmoud ALE, Yagi SM. 2011. Ethnobotanical study of medicinal plants in the Blue Nile State, South-eastern Sudan. Journal of Medicinal Plants Research 5(17):4287-4297.

Mustafa B, Hajdari A, Krasniqi F, Hoxha E, Ademi H, Quave C L, Pieroni A. 2012. Medical ethnobotany of the Albanian Alps in Kosovo. Journal of Ethnobiology and Ethnomedicine 8(1):6.

Oliveira ES, Torres DF, Brooks SE, Alves RR. 2010. The medicinal animal markets in the metropolitan region of Natal City, northeastern Brazil. Journal of Ethnopharmacology 130(1):54-60.

Parthiban R, Vijayakumar S, Prabhu S, Esther JG, Yabesh M. 2016. Quantitative traditional knowledge of medicinal plants used to treat livestock diseases from Kudavasal taluk of Thiruvarur district, Tamil Nadu, India. Brazilian Journal of Pharmacognosy 26:109-121.

Ragupathy S, Steven NG, Maruthakkutti M, Velusamy B, UI-Huda MM. 2008. Consensus of the 'Malasars' traditional aboriginal knowledge of medicinal plants in the Velliangiri holy hills, India. Journal of Ethnobiology and Ethnomedicine 4(1):8.

Rahman AHMM. 2009. Taxonomic Studies on the Family Asteraceae (Compositae) of the Rajshahi division. Ph.D. thesis, Department of Botany, University of Rajshahi, Bangladesh.

Raunkiær C. 1934. The life forms of plants and statistical plant geography. Clarendon Press, Oxford, U.K.

Raunkiær C. 1937. Plant life forms. Oxford University Press, Oxford, U.K.
Rehman MN, Ahmad M, Sultana S, Zafar M, Edward S. 2017. Relative popularity level of medicinal plants in Talagang, Punjab Province, Pakistan. Brazilian Journal of Pharmacognosy 27:751-775

Shanmugam S, Gayathri N, Sakthivel B, Ramar S, Rajendran K. 2009. Plants used as Medicine by Paliyar Tribes of Shenbagathope in Virudhunagar District of Tamilnadu, India. Ethnobotanical Leaflets 13:370-378

Sharma NK. 2016. Traditional Ethnomedicinal Knowledge of Indian Tribes. Current Science 110(4):486-487.

Siddiqui TO, Javed K, Aslam MM. 2000. Folk medicinal claims of Western Uttar Pradesh, India. Hamdard Medicus 43:59-60.

Sinha MK. 2017. Ethnomedicinal plants used in Tribes of Bharatpur block (Koriya district) C.G. Journal of Medicinal Plants Studies 5(4):17-21.

Stevens PF (2001 onwards). Angiosperm Phylogeny Website Version 14, July 2017. Retrieved from http://www.mobot.org/MOBOT/research/APweb/.

Teklehaymanot T. 2009. Ethnobotanical study of knowledge and medicinal plants use by the people in Dek Island in Ethiopia. Journal of Ethnopharmacology 124(1):69-78.

Trotter R, Logan M. 1986. Informant consensus: a new approach for identifying potentially effective medicinal plants. In Plants in Indigenous Medicine and Diet: Biobehavioral Approaches. Edited by NL Etkin. Bedford Hills, Redgrave Publishers, NY, US, Pp. 91-112.

Venkatachalapathi A, Sangeeth T, Ajmal Ali M, Tamilselvi SS, Paulsamy S, Al-Hemaidc FMA. 2018. Ethnomedicinal assessment of Irula tribes of Walayar valley of Southern Western Ghats, India. Saudi Journal of Biological Sciences 25:760-775.

Vijayakumar S, Harikrishnan JB, Prabhu S, Morvin Yabesh JE, Manogar P. 2016. Quantitative Ethnobotanical Survey of Traditional Siddha Medical Practitioners from Thiruvarur District with Hepatoprotective Potentials through In Silico Methods. Achievements in the Life Sciences 10(1):11-26.

Wagh VV, Jain AK. 2014. Ethnomedicinal Uses of Underground Plant Parts in Jhabua District of Madhya Pradesh, India. Advances in Biological Research 8(4):151-156.

Wanjohi BK, Njenga EW, Sudoi V, Kipkore WK, Moore HL and Davies MJ. 2020. Ecological Knowledge of indigenous plants among the Marakwet Community (Embobut Basin), Elgeyo Marakwet County (Kenya). Ethnobotany Research and Applications 20:1-16.

Weckerle CS, de Boer HJ, Puri RK, van Andel T, Bussmann RW, Leonti M. 2018. Recommended 
standards for conducting and reporting ethnopharmacological field studies. Journal of Ethnopharmacology 210:125-132.

World Health Organization (WHO). 2012. World Malaria Report. Available at: https://www.who.int/malaria/publications/world_mala ria_report_2012/en/

Yadav R, Khare RK. 2014. Traditional Uses of Medicinal Plants among the Tribals of Shivpuri District (M.P.). Periodic Research III (II):70-75. 